\title{
Global Behaviors of a Class of Discrete SIRS Epidemic Models with Nonlinear Incidence Rate
}

\author{
Lei Wang, ${ }^{1,2}$ Zhidong Teng, ${ }^{2}$ and Long Zhang ${ }^{2}$ \\ ${ }^{1}$ Department of Medical Engineering and Technology, Xinjiang Medical University, Urumqi 830011, China \\ ${ }^{2}$ College of Mathematics and Systems Science, Xinjiang University, Urumqi 830046, China \\ Correspondence should be addressed to Zhidong Teng; zhidong@xju.edu.cn
}

Received 3 November 2013; Accepted 3 February 2014; Published 17 March 2014

Academic Editor: Yun Kang

Copyright (c) 2014 Lei Wang et al. This is an open access article distributed under the Creative Commons Attribution License, which permits unrestricted use, distribution, and reproduction in any medium, provided the original work is properly cited.

\begin{abstract}
We study a class of discrete SIRS epidemic models with nonlinear incidence rate $F(S) G(I)$ and disease-induced mortality. By using analytic techniques and constructing discrete Lyapunov functions, the global stability of disease-free equilibrium and endemic equilibrium is obtained. That is, if basic reproduction number $\mathscr{R}_{0}<1$, then the disease-free equilibrium is globally asymptotically stable, and if $\mathscr{R}_{0}>1$, then the model has a unique endemic equilibrium and when some additional conditions hold the endemic equilibrium also is globally asymptotically stable. By using the theory of persistence in dynamical systems, we further obtain that only when $\mathscr{R}_{0}>1$, the disease in the model is permanent. Some special cases of $F(S) G(I)$ are discussed. Particularly, when $F(S) G(I)=\beta S I /(1+\lambda I)$, it is obtained that the endemic equilibrium is globally asymptotically stable if and only if $\mathscr{R}_{0}>1$. Furthermore, the numerical simulations show that for general incidence rate $F(S) G(I)$ the endemic equilibrium may be globally asymptotically stable only as $\mathscr{R}_{0}>1$.
\end{abstract}

\section{Introduction}

During the past decades, no matter discrete epidemic models or continuous epidemic models, have been widely studied. Many important and interesting results can be found in [1-28] and the references cited therein. The main research subjects are the computation of the threshold value or basic reproduction number which distinguishes whether the infectious disease will persist or die out, the local and global stability of the disease-free equilibrium and endemic equilibrium, the extinction, persistence, and permanence of the disease, and the bifurcations, chaos, and more complex dynamical behaviors of the models.

Among these questions, global stability of equilibria has always been one of the research focuses and difficult problems. Many authors have investigated this question using the second Lyapunov method (see [29]). The most popular types of Lyapunov functions candidate for population biology models are the Volterra-type functions $x-x^{*}-\ln \left(x / x^{*}\right)$ and the quadratic function $(c / 2)\left(x-x^{*}\right)^{2}$. The former has been successfully applied for various disease propagation models by Korobeinikov and his coworkers (see [7-10] and the references cited therein). In [11], Li et al. presented an algebraic approach to prove the global stability, which can provide the method of constructing a Lyapunov function and prove the negative definiteness of the derivative. Recently, by combining Volterra functions and quadratic functions, Vargas-De-León has studied global stability of classic continuous SIS, SIR, and SIRS epidemic models with constant recruitment, disease-induced death, and standard incidence rate and bilinear incidence rate in [12, 13], respectively. McCluskey in [14-16] introduced the Lyapunov functional formed as $\int_{0}^{\tau}\left(x(t-s) / x^{*}-1-\ln \left(x(t-s) / x^{*}\right)\right) d s$ to investigate global stability of endemic equilibrium of SEIR epidemic model with distributed delay or discrete delay.

It is well known that a crucial role in mathematical models of infectious disease is played by the so-called incidence rate, namely, a function describing the mechanism of transmission of the disease. In most epidemiological models, bilinear incidence rate $\beta S I$ and standard incidence rate $\beta S I / N$ are frequently used, where $N$ is the total number of the population $(N=S+I+R)$ and $\beta>0$ is the per capita 
contact rate. These incidences imply that the contact number between $S$ and $I$ is proportional to $S I$ or $S I / N$. But the infection probability per contact is likely influenced by the number of infective and susceptible individuals, because more infective individuals can increase the infection risk and susceptible individuals would avoid the contact with infective individuals. Therefore, a number of nonlinear incidence rates are suggested by researchers. After studying the cholera epidemic spread in Bari in 1973, Capasso and Serio [17] introduced the saturated incidence rate $\beta S G(I)$ into epidemic models. To incorporate the effect of the behavioral changes of the susceptible individuals, Liu et al. proposed the general incidence rate $\beta S I^{p} /\left(1+k I^{q}\right)$ in [18], where $p, q>0$ and $k \geq 0$. The special cases when $p$ and $q$ are given different values have been used by many authors (see, e.g., Korobeinikov and Maini [6], Ruan and Wang [19], and Xiao and Ruan [20]).

However, until now, to the best of our knowledge, there are few search results about global stability of equilibria for discrete SIRS model with nonlinear incidence rate. $\mathrm{Hu}$ et al. in [28] discussed local stability and complex dynamical behaviors for a class of discrete SIRS epidemic models with general nonlinear incidence rate discretized by the forward Euler scheme. Enatsu et al. in [22] proposed a class of discrete SIR epidemic models with bilinear incidence rate, which are derived from continuous SIR epidemic models with distributed delays by using a variation of the backward Euler method, and obtained that global stability of diseasefree equilibrium and endemic equilibrium. Muroya et al. in [23] discussed global stability and permanence of a discrete epidemic model with bilinear incidence rate and for disease with immunity and latency spreading in a heterogeneous host population, which is also discretized from the continuous case by using the backward Euler method. In [24], Enatsu et al. studied a class of discrete SIR epidemic models with nonlinear incidence rates and distributed delays, which are derived from the corresponding continuous SIR epidemic models by applying a variation of the backward Euler discretization. Using discrete-time analogue of Lyapunov functionals, the global asymptotic stability of the diseasefree equilibrium and endemic equilibrium is fully determined by the basic reproduction number $R_{0}$, when the infection incidence rate has a suitable monotone property.

Motivated by the fact that discrete epidemic models are more appropriate approach to understand disease transmission dynamics and to evaluate eradication policies because they permit arbitrary time step units, preserving the basic features of corresponding continuous models, in this paper, we will extend a discrete-time analogue of Lyapunov techniques proposed in [25-27] to the following discrete SIRS epidemic models with nonlinear incidence rate $F(S) G(I)$, which is established by using the backward Euler scheme (see $[30,31]$ ) to discretize the corresponding continuous SIRS epidemic model:

$$
\begin{aligned}
S(n+1)= & S(n)+\Lambda-F(S(n+1)) G(I(n+1)) \\
& -\mu S(n+1)+\gamma R(n+1)
\end{aligned}
$$

$$
\begin{gathered}
I(n+1)=I(n)+F(S(n+1)) G(I(n+1)) \\
-(k+\mu+\alpha) I(n+1), \\
R(n+1)=R(n)+k I(n+1)-(\mu+\gamma) R(n+1) .
\end{gathered}
$$

We will investigate the global behaviors of solutions of model (1). By constructing new discrete Lyapunov functions, we will establish some new criteria on the global asymptotic stability of the disease-free equilibrium and endemic equilibrium for model (1). By using the theory of persistence in dynamical systems, we will further obtain the sufficient and necessary conditions for the permanence of the disease for model (1).

The organization of this paper is as follows. In Section 2, the existence of equilibria and positivity of solutions for model (1) are given. In Section 3, the results on the global asymptotic stability of the disease-free equilibrium and endemic equilibrium for model (1) are stated and proved. In Section 4, the results on the permanence of the disease in model (1) are established. In Section 5, the global asymptotic stability of the endemic equilibrium of model (1) for the special case $F(S)=S /(1+\lambda S)$ is discussed. Finally, some examples are given to illustrate the main theoretical results in Section 6.

\section{Equilibria and Positivity}

For model (1), $S(n), I(n)$, and $R(n)$ represent the numbers of susceptible, infectious, and recovered individuals at $n$th generation, respectively. The parameters $\Lambda, \mu, \alpha$, and $k$ are positive constants and $\gamma$ is nonnegative constant in which $\Lambda$ is the recruitment rate into the population, $\mu$ is the natural death rate, $\alpha$ is the disease-induced death rate, $k$ is the recovery rate of the infectious individuals, $\gamma$ is the rate of losing immunity, $\gamma>0$ implies that the recovered individuals would lose the immunity, and $\gamma=0$ implies that the recovered individuals acquire permanent immunity. The spread of disease can be described by general form with incidence rate $F(S) G(I)$; that is, the incidence rate depends on the number of the susceptible individuals and the number of the infectious individuals. This generalizes the bilinear incidence rate (i.e., $F(S) G(I)=\beta S I)$, saturated incidence rate with respect to $S$ (i.e., $F(S) G(I)=\beta S I /(1+\lambda S)$ ), and saturated incidence rate with respect to $I$ (i.e., $F(S) G(I)=\beta S I /(1+\omega I)$ ), where $\beta>0$, $\lambda \geq 0$, and $\omega \geq 0$ are constants, which denotes the contact coefficient and the saturated coefficient, respectively.

The initial condition for model (1) is given by

$$
S(0)>0, \quad I(0)>0, \quad R(0) \geq 0 .
$$

In this paper, for functions $F(S)$ and $G(I)$, we firstly introduce the following assumption.

$\left(H_{1}\right) F(S)$ and $G(I)$ are positive, monotonically increasing, and continuous differentiable functions defined for all $S \geq 0$ and $I \geq 0$, the derivative $G^{\prime}(0)$ exists, and $F(0)=$ $G(0)=0$. Furthermore, $G(I) / I$ is nonincreasing for all $I>0$. 
Remark 1. Assumption $\left(H_{1}\right)$ is basic for model (1). In fact, for many special cases of $F(S) G(I)$, for example, $F(S) G(I)=\beta S I$, $F(S) G(I)=\beta S I /(1+\lambda S)$, and $F(S) G(I)=\beta S I /(1+\omega I),\left(H_{1}\right)$ is always satisfied.

In order to obtain the existence of disease-free equilibrium and endemic equilibrium of model (1), we introduce a constant

$$
\mathscr{R}_{0}=\frac{F(\Lambda / \mu) G^{\prime}(0)}{k+\mu+\alpha} .
$$

We have the following result.

Theorem 2. Assume that $\left(H_{1}\right)$ holds.

(1) When $\mathscr{R}_{0} \leq 1$, then model (1) has only a unique disease-free equilibrium $E^{0}(\Lambda / \mu, 0,0)$.

(2) When $\mathscr{R}_{0}>1$, then model (1) shows a unique endemic equilibrium $E^{*}\left(S^{*}, I^{*}, R^{*}\right)$, except for $E^{0}$, where $S^{*}, I^{*}$, and $R^{*}$ satisfy

$$
\begin{aligned}
& \Lambda=F\left(S^{*}\right) G\left(I^{*}\right)+\mu S^{*}-\gamma R^{*}, \\
& I^{*}(k+\mu+\alpha)=F\left(S^{*}\right) G\left(I^{*}\right), \\
& k I^{*}=(\mu+\gamma) R^{*} .
\end{aligned}
$$

Proof. Obviously, model (1) always has a disease-free equilibrium $E^{0}(\Lambda / \mu, 0,0)$. From (4), we have

$$
R^{*}=\frac{k I^{*}}{\mu+\gamma}, \quad \Lambda=I^{*}(k+\mu+\alpha)+\mu S^{*}-\gamma R^{*} .
$$

Hence,

$$
\begin{aligned}
S^{*} & =\frac{1}{\mu}\left(\Lambda-I^{*}\left(k+\mu+\alpha-\frac{k \gamma}{\mu+\gamma}\right)\right) \\
& =\frac{\Lambda}{\mu}-I^{*} \frac{(\mu+\alpha)(\mu+\gamma)+k \mu}{\mu(\mu+\gamma)},
\end{aligned}
$$

and from the second equation of (4) we further have

$$
I^{*}(k+\mu+\alpha)=F\left(\frac{\Lambda}{\mu}-I^{*} \frac{(\mu+\alpha)(\mu+\gamma)+k \mu}{\mu(\mu+\gamma)}\right) G\left(I^{*}\right) .
$$

When $I>0$, let

$$
H(I)=k+\mu+\alpha-F\left(\frac{\Lambda}{\mu}-I \frac{(\mu+\alpha)(\mu+\gamma)+k \mu}{\mu(\mu+\gamma)}\right) \frac{G(I)}{I} .
$$

Then by $\left(H_{1}\right)$ we obtain

$$
\lim _{I \rightarrow 0^{+}} H(I)=k+\mu+\alpha-F\left(\frac{\Lambda}{\mu}\right) G^{\prime}(0) \begin{cases}\geq 0, & \mathscr{R}_{0} \leq 1, \\ <0, & \mathscr{R}_{0}>1 .\end{cases}
$$

Let $\bar{I}=\Lambda(\mu+\gamma) /((\mu+\gamma)(\mu+\alpha)+k \mu)$; then we obviously have $H(\bar{I})=k+\mu+\alpha>0$. From $\left(H_{1}\right), F((\Lambda / \mu)-I(((\mu+$ $\alpha)(\mu+\gamma)+k \mu) / \mu(\mu+\gamma)))$ is monotonically decreasing for $I \in(0, \bar{I}]$, and hence $H(I)$ is monotonically increasing for $I \in$ $(0, \bar{I}]$. Thus, from (9), we obtain that when $\mathscr{R}_{0} \leq 1$ equation $H(I)=0$ has not any solution in $(0, \bar{I})$ and when $\mathscr{R}_{0}>1$ equation $H(I)=0$ has a unique positive solution $I^{*}$ in $(0, \bar{I})$. This shows that when $\mathscr{R}_{0} \leq 1$ model (1) does not have any endemic equilibrium. When $\mathscr{R}_{0}>1$, let

$$
R^{*}=\frac{k I^{*}}{\mu+\gamma}, \quad S^{*}=\frac{1}{\mu}\left(\Lambda-I^{*}\left(k+\mu+\alpha-\frac{k \gamma}{\mu+\alpha}\right)\right),
$$

and then $E^{*}\left(S^{*}, I^{*}, R^{*}\right)$ is a unique endemic equilibrium of model (1). This completes the proof.

From Theorem 2, we can claim that the basic reproduction number of model (1) is $\mathscr{R}_{0}$. On the positivity and ultimate boundedness of solutions of model (1), we obtain the following theorem.

Theorem 3. Assume that $\left(H_{1}\right)$ holds. Let $(S(n), I(n), R(n))$ be the solution of model (1) with initial conditions (2); then $(S(n), I(n), R(n))$ is positive for any $n>0$ and ultimately bounded.

Proof. Let $(S(n), I(n), R(n))$ be any solution of model (1) with initial conditions (2). Further, let $N(n)=S(n)+I(n)+R(n))$; then model (1) is equivalent to the following form:

$$
\begin{gathered}
I(n+1)=\frac{1}{1+k+\mu+\alpha} \\
\times(I(n)+F(N(n+1) \\
\quad-I(n+1)-R(n+1)) \\
\quad \times \quad(I(n+1))), \\
R(n+1)=\frac{R(n)+k I(n+1)}{1+\mu+\gamma}, \\
N(n+1)=\frac{N(n)+\Lambda-\alpha I(n+1)}{1+\mu},
\end{gathered}
$$

$$
\begin{aligned}
S(n+1)= & S(n)+\Lambda-F(S(n+1)) G(I(n+1)) \\
& -\mu S(n+1)+\gamma R(n+1) .
\end{aligned}
$$


In the following, we will use the induction to prove the positivity of $(S(n), I(n), R(n))$. When $n=0$, we have

$$
\begin{aligned}
I(1)= & \frac{1}{1+k+\mu+\alpha} \\
& \times(I(0)+F(N(1)-I(1)-R(1)) G(I(1))), \\
R(1)= & \frac{R(0)+k I(1)}{1+\mu+\gamma}, \quad N(1)=\frac{N(0)+\Lambda-\alpha I(1)}{1+\mu}, \\
S(1)= & S(0)+\Lambda-F(S(1)) G(I(1))-\mu S(1)+\gamma R(1) .
\end{aligned}
$$

From (13)-(15) we see that as long as $I(1)$ is confirmed, then $R(1), N(1)$, and $S(1)$ will be whereafter confirmed.

Firstly, we prove that if $I(1)>0$, then $S(1)>0$ and $R(1)>$ 0 . From (14), we directly obtain $R(1)>0$ when $I(1)>0$. Let $x=S(1)$, and from (15) we obtain

$$
\Phi(x) \triangleq(1+\mu) x+F(x) G(I(1))-\gamma R(1)-S(0)-\Lambda=0 .
$$

It is obvious that, when $I(1)>0, \Phi(x)$ is monotonically increasing for $x \geq 0$. Obviously, $\Phi(x)$ is a continuous function for $x$. Since $\Phi(0)=-\gamma R(1)-S(0)-\Lambda<0$ and $\lim _{x \rightarrow+\infty} \Phi(x)=+\infty$, we obtain that $\Phi(x)=0$ has a unique positive solution $\bar{x}$. Therefore, we further have $S(1)=\bar{x}>0$. Furthermore, we also have $N(1)=S(1)+I(1)+R(1)>0$.

Let $y=I(1)$ ); then from (13) we see that $y$ must satisfy the following equation:

$$
\begin{aligned}
\Psi(y) \triangleq & y-\frac{1}{1+k+\mu+\alpha} \\
& \times(I(0)+F(N(1)-y-R(1)) G(y))=0,
\end{aligned}
$$

where

$$
N(1)=\frac{N(0)+\Lambda-\alpha y}{1+\mu}, \quad R(1)=\frac{R(0)+k y}{1+\mu+\gamma}
$$

Denote

$$
\begin{aligned}
& a_{0}=\frac{N(0)+\Lambda}{1+\mu}-\frac{R(0)}{1+\mu+\gamma}, \\
& b_{0}=\frac{\alpha}{1+\mu}+1+\frac{k}{1+\mu+\gamma}
\end{aligned}
$$

Obviously, $a_{0}>0$. Let $y_{0}=a_{0} / b_{0}$; then when $y=y_{0}$ we have $N(1)-y-R(1)=0$. We also have that $N(1)-y-R(1)$ is monotonically decreasing with respect to $y \in\left[0, y_{0}\right]$. Hence, by $\left(H_{1}\right), F(N(1)-y-R(1))$ is also monotonically decreasing with respect to $y \in\left[0, y_{0}\right]$. From the expression of $\Psi(y)$ and $\left(H_{1}\right)$, we obtain that $\Psi(y)$ is monotonically increasing for $y \in\left[0, y_{0}\right]$. Obviously, $\Psi(y)$ is a continuousfunction for $y$.
Since

$$
\begin{gathered}
\Psi(0)=-\frac{I(0)}{1+k+\mu+\alpha}<0, \\
\Psi\left(y_{0}\right)=y_{0}-\frac{1}{1+k+\mu+\alpha} I(0) \\
=\frac{(\Lambda+N(0))(1+\mu+\gamma)-R(0)(1+\mu)}{\alpha(1+\mu+\gamma)+(1+\mu)(1+\mu+\gamma)+k(1+\mu)} \\
\geq \frac{1+S(0)+I(0)}{1+k+\mu+\alpha} I(0) \\
=\frac{\Lambda+S(0)}{1+k+\mu+\alpha},
\end{gathered}
$$

there exists a unique $\bar{y} \in\left(0, y_{0}\right)$ such that $\Psi(\bar{y})=0$.

Now, we show that $\bar{y}$ is a unique solution of $\Psi(y)=0$ on $(0, \infty)$. Otherwise, there is a $y^{\prime} \in\left[y_{0}, \infty\right)$ such that $\Psi\left(y^{\prime}\right)=0$. Since $y^{\prime} \geq y_{0}$, we have $N(1)-y-R(1) \leq 0$ when $y=y^{\prime}$. From $\left(H_{1}\right)$, we have $F(S) \geq 0$ for any $S \geq 0$; hence from $\Psi\left(y^{\prime}\right)=0$ we further have $y^{\prime} \leq I(0) /(1+\mu+\alpha+k)$. On the other hand, since $a_{0}>I(0) /(1+\mu)$ and $b_{0}<(1+\mu+\alpha+k) /(1+\mu)$, we obtain $y^{\prime}>I(0) /(1+\mu+\alpha+k)$, which leads to a contradiction.

Therefore, we certainly have $I(1)=\bar{y}>0$. From the above discussions, we finally have $I(1)>0, S(1)>0$, and $R(1)>0$.

When $n=1$, we obtain

$$
\begin{gathered}
I(2)=\frac{1}{1+k+\mu+\alpha} \\
\times(I(1)+F(N(1)-I(1)-R(1)) G(I(1))), \\
R(2)=\frac{R(1)+k I(2)}{1+\mu+\gamma}, \\
N(2)=\frac{N(1)+\Lambda-\alpha I(2)}{1+\mu}, \\
S(2)=S(1)+\Lambda-F(N(2)-I(2)-R(2)) \\
\times G(I(2))-\mu S(2)+\gamma R(2) .
\end{gathered}
$$

Obviously, using a similar argument in the above process, we also can obtain $S(2)>0, I(2)>0$, and $R(2)>0$. Lastly, by using the induction, we can finally obtain $S(n)>0, I(n)>0$, and $R(n)>0$ for all $n>0$.

From the third equation of model (11), we have

$$
N(n+1) \leq \frac{1}{1+\mu}(N(n)+\Lambda) .
$$

Since comparison equation,

$$
U(n+1)=\frac{1}{1+\mu}(U(n)+\Lambda),
$$


has a globally asymptotically stable equilibrium $U^{*}=\Lambda / \mu$, from the comparison principle of difference equations (see [32]), we finally obtain

$$
\limsup _{n \rightarrow \infty} N(n) \leq \frac{\Lambda}{\mu}
$$

Therefore, $(S(n), I(n), R(n))$ is also ultimately bound. This completes the proof.

\section{Global Stability}

Now, we are concerned with the global asymptotic stability of disease-free equilibrium $E^{0}$ and endemic equilibrium $E^{*}$ of model (1), respectively.

Theorem 4. Assume that $\left(H_{1}\right)$ holds. Then disease-free equilibrium $E^{0}(\Lambda / \mu, 0,0)$ of model (1) is globally asymptotically stable if $\mathscr{R}_{0}<1$ and is globally attractive if $\mathscr{R}_{0}=1$.

Proof. Calculating the linearization system of model (1) at equilibrium $E^{0}$, we have

$$
\begin{gathered}
u_{n+1}=u_{n}-F\left(\frac{\Lambda}{\mu}\right) G^{\prime}(0) v_{n+1}-\mu u_{n+1}+\gamma w_{n+1}, \\
v_{n+1}=v_{n}+F\left(\frac{\Lambda}{\mu}\right) G^{\prime}(0) v_{n+1}-(k+\mu+\alpha) v_{n+1}, \\
w_{n+1}=w_{n}+k v_{n+1}-(\mu+\gamma) w_{n+1} .
\end{gathered}
$$

From the second equation of system (25), we have

$$
v_{n+1}=\frac{v_{n}}{1+k+\mu+\alpha-F(\Lambda / \mu) G^{\prime}(0)} .
$$

When $\mathscr{R}_{0}<1$, we obtain

$$
\begin{aligned}
0 & <\frac{1}{1+k+\mu+\alpha-F(\Lambda / \mu) G^{\prime}(0)} \\
& =\frac{1}{1+(k+\mu+\alpha)\left(1-\mathscr{R}_{0}\right)}<1 .
\end{aligned}
$$

Therefore, $\lim _{n \rightarrow \infty} v_{n}=0$. By

$$
u_{n+1}=\frac{u_{n}-F(\Lambda / \mu) G^{\prime}(0) v_{n+1}}{1+\mu}, \quad w_{n+1}=\frac{w_{n}+k v_{n+1}}{1+\mu+\gamma},
$$

we further obtain $\lim _{n \rightarrow \infty} u_{n}=0$ and $\lim _{n \rightarrow \infty} w_{n}=0$. This shows that $E^{0}$ is locally stable when $\mathscr{R}_{0}<1$. Since the case $\mathscr{R}_{0}=1$ is a critical one for model (1), in the following, we discuss global attractivity of disease-free equilibrium $E^{0}$ when $\mathscr{R}_{0} \leq 1$.

Let $(S(n), I(n), R(n))$ be any positive solution of model (1) with initial conditions (2). We need to consider the following two cases.

Case $1 . N(n) \geq \Lambda / \mu$ for all $n=1,2, \ldots$.

Case 2. There exists an integer $n_{1}>0$ such that $N\left(n_{1}\right)<\Lambda / \mu$.
For Case 1, from (24), we directly have

$$
\lim _{n \rightarrow \infty} N(n)=\frac{\Lambda}{\mu}
$$

From third equation of (11), we further obtain

$$
\begin{aligned}
\lim _{n \rightarrow \infty} I(n) & =\lim _{n \rightarrow \infty} \frac{1}{\alpha}[N(n)(1+\mu)-N(n-1)-\Lambda] \\
& =\frac{1}{\alpha}\left[\frac{\Lambda}{\mu}(1+\mu)-\frac{\Lambda}{\mu}-\Lambda\right]=0 .
\end{aligned}
$$

For Case 2, by using the iterative computations to inequality (22), we can obtain $N(n)<\Lambda / \mu$ for all $n \geq n_{1}$. Hence, $S(n)<\Lambda / \mu$ for all $n \geq n_{1}$. From $\left(H_{1}\right)$, we further obtain

$$
F(S(n+1))<F\left(\frac{\Lambda}{\mu}\right), \quad \forall n \geq n_{1}
$$

Since

$$
\frac{G(I(n+1))}{I(n+1)} \leq \lim _{I \rightarrow 0^{+}} \frac{G(I)}{I}=G^{\prime}(0),
$$

from the second equation of model (1), it follows that, for all $n \geq n_{1}$,

$$
\begin{aligned}
I(n+1)-I(n)= & F(S(n+1)) G(I(n+1)) \\
& -(k+\mu+\alpha) I(n+1) \\
= & I(n+1)(F(S(n+1)) \\
& \left.\times \frac{G(I(n+1))}{I(n+1)}-(k+\mu+\alpha)\right) \\
\leq & I(n+1)\left(F\left(\frac{\Lambda}{\mu}\right) G^{\prime}(0)-(k+\mu+\alpha)\right) \\
= & (k+\mu+\alpha) I(n+1)\left(\mathscr{R}_{0}-1\right) .
\end{aligned}
$$

If $\mathscr{R}_{0} \leq 1$, then

$$
I(n+1)-I(n) \leq 0 \text { for all } n \geq n_{1} .
$$

Hence, $I(n)$ is nonincreasing for $n \geq n_{1}$. Consequently, $\lim _{n \rightarrow \infty} I(n)=\widehat{I}$ exists and $\widehat{I} \geq 0$.

Suppose $\widehat{I}>0$; then from the second and third equations of model (11), we can obtain that $\lim _{n \rightarrow \infty} R(n)$ and $\lim _{n \rightarrow \infty} N(n)$ exist, and

$$
\lim _{n \rightarrow \infty} R(n)=\frac{k \widehat{I}}{\mu+\gamma}:=\widehat{R}, \quad \lim _{n \rightarrow \infty} N(n)=\frac{\Lambda-\alpha \widehat{I}}{\mu}:=\widehat{N} .
$$

From $S(n)=N(n)-I(n)-R(n)$, it follows that $\lim _{n \rightarrow \infty} S(n)=$ $\widehat{S}$ exists. Obviously, we have $\widehat{R}>0, \widehat{N} \geq 0$, and $\widehat{S} \geq 0$. 
Taking $n \rightarrow \infty$ from the both sides of model (1), we can obtain the following equations:

$$
\begin{gathered}
\Lambda-F(\widehat{S}) G(\widehat{I})-\mu \widehat{S}+\gamma \widehat{R}=0, \\
\widehat{I}(k+\mu+\alpha)-F(\widehat{S}) G(\widehat{I})=0, \\
k \widehat{I}-(\mu+\gamma) \widehat{R}=0 .
\end{gathered}
$$

Hence, $(\widehat{S}, \widehat{I}, \widehat{R})$ is an equilibrium of model (1). However, from Theorem 2 , we see that when $\mathscr{R}_{0} \leq 1$, (36) only has a unique solution $\widehat{S}=\Lambda / \mu, \widehat{I}=0$, and $\widehat{R}=0$. This leads to a contradiction. Therefore, we have $\widehat{I}=0$.

Therefore, we always have $\lim _{n \rightarrow \infty} I(n)=\widehat{I}=0$. By (35), it follows that $\lim _{n \rightarrow \infty} R(n)=0$ and $\lim _{n \rightarrow \infty} N(n)=\Lambda / \mu$. Consequently, $\lim _{n \rightarrow \infty} S(n)=\Lambda / \mu$. This shows that diseasefree equilibrium $E^{0}=(\Lambda / \mu, 0,0)$ is globally attractive when $\mathscr{R}_{0} \leq 1$. This completes the proof.

In order to obtain the global asymptotic stability of endemic equilibrium $E^{*}$ of model (1), we need the following assumptions.

$\left(H_{2}\right)$ For any $S>0$,

$$
\frac{F(S)}{F(S)-F\left(S^{*}\right)}-\frac{\left(S^{*}\right)^{2}}{F\left(S^{*}\right)\left(S-S^{*}\right)} \geq 0 .
$$

$\left(H_{3}\right)$ For any $S>0$,

$$
-\frac{\mu\left(S^{*}\right)^{2}}{F\left(S^{*}\right)}+\gamma R^{*}\left(\frac{F(S)}{F(S)-F\left(S^{*}\right)}-\frac{\left(S^{*}\right)^{2}}{F\left(S^{*}\right)\left(S-S^{*}\right)}\right) \leq 0 .
$$

Theorem 5. Assume that $\left(H_{1}\right)-\left(H_{3}\right)$ hold. If $\mathscr{R}_{0}>1$, then endemic equilibrium $E^{*}\left(S^{*}, I^{*}, R^{*}\right)$ of model (1) is globally asymptotically stable.

Proof. We firstly define the auxiliary functions as follows:

$$
\begin{gathered}
V_{1}(S)=S-S^{*}-\int_{S^{*}}^{S} \frac{F\left(S^{*}\right)}{F(\eta)} d \eta, \\
V_{2}(I)=I-I^{*}-\int_{I^{*}}^{I} \frac{G\left(I^{*}\right)}{G(\eta)} d \eta, \\
V_{3}(R)=\frac{1}{2}\left(R-R^{*}\right)^{2}, \\
V_{4}(N, R)=\frac{1}{2}\left(N-N^{*}+\frac{\alpha}{k}\left(R-R^{*}\right)\right)^{2},
\end{gathered}
$$

where $N=S+I+R$ and $N^{*}=S^{*}+I^{*}+R^{*}$. From $\left(H_{1}\right)$, we easily obtain that when $S \neq S^{*}$

$$
V_{1}(S)>S-S^{*}-\int_{S^{*}}^{S} \frac{F\left(S^{*}\right)}{F\left(S^{*}\right)} d \eta=0
$$

and when $I \neq I^{*}$

$$
V_{2}(I)>I-I^{*}-\int_{I^{*}}^{I} \frac{G\left(I^{*}\right)}{G\left(I^{*}\right)} d \eta=0 .
$$

Let $(S(n), I(n), R(n))$ be any positive solution of model (1) with initial condition (2). By computing $\Delta V_{1}(n)=V_{1}(S(n+$ 1)) $-V_{1}(S(n))$, we have

$$
\Delta V_{1}(n)=S(n+1)-S(n)-\int_{S(n)}^{S(n+1)} \frac{F\left(S^{*}\right)}{F(\eta)} d \eta
$$

From $\left(H_{1}\right)$, it follows that

$$
\begin{aligned}
&-\frac{F\left(S^{*}\right)}{F(\eta)} \leq-\frac{F\left(S^{*}\right)}{F(S(n+1))} \quad \text { if } S(n+1) \geq \eta \geq S(n), \\
&-\frac{F\left(S^{*}\right)}{F(\eta)} \geq-\frac{F\left(S^{*}\right)}{F(S(n+1))} \quad \text { if } S(n+1) \leq \eta \leq S(n) .
\end{aligned}
$$

Hence,

$$
\begin{aligned}
& -\int_{S(n)}^{S(n+1)} \frac{F\left(S^{*}\right)}{F(\eta)} d \eta \leq-\frac{F\left(S^{*}\right)}{F(S(n+1))}(S(n+1)-S(n)), \\
& \Delta V_{1}(n) \leq S(n+1)-S(n)-\frac{F\left(S^{*}\right)}{F(S(n+1))} \\
& \times(S(n+1)-S(n)) \\
& =\left(1-\frac{F\left(S^{*}\right)}{F(S(n+1))}\right) \\
& \times(\Lambda-F(S(n+1)) G(I(n+1)) \\
& -\mu S(n+1)+\gamma R(n+1)) \\
& =\left(1-\frac{F\left(S^{*}\right)}{F(S(n+1))}\right) \\
& \times\left(\mu S^{*}-\mu S(n+1)+F\left(S^{*}\right) G\left(I^{*}\right)\right. \\
& -F(S(n+1)) G(I(n+1)) \\
& \left.+\gamma R(n+1)-\gamma R^{*}\right) \\
& =-\mu\left(1-\frac{F\left(S^{*}\right)}{F(S(n+1))}\right)\left(S(n+1)-S^{*}\right) \\
& +(k+\mu+\alpha) I^{*} \\
& \times\left(1-\frac{F\left(S^{*}\right)}{F(S(n+1))}\right)
\end{aligned}
$$




$$
\begin{aligned}
& \times\left(1-\frac{F(S(n+1)) G(I(n+1))}{F\left(S^{*}\right) G\left(I^{*}\right)}\right) \\
& +\gamma\left(1-\frac{F\left(S^{*}\right)}{F(S(n+1))}\right)\left(R(n+1)-R^{*}\right) \\
& =-\mu\left(1-\frac{F\left(S^{*}\right)}{F(S(n+1))}\right)\left(S(n+1)-S^{*}\right) \\
& +\gamma\left(1-\frac{F\left(S^{*}\right)}{F(S(n+1))}\right)\left(R(n+1)-R^{*}\right) \\
& +(k+\mu+\alpha) I^{*} \\
& \times\left(1-\frac{F\left(S^{*}\right)}{F(S(n+1))}\right. \\
& +\frac{F(S(n+1)) G(I(n+1))}{F\left(S^{*}\right) G\left(I^{*}\right)} \\
& \left.+\frac{G(I(n+1))}{G\left(I^{*}\right)}\right) .
\end{aligned}
$$
have

By computing $\Delta V_{2}(n)=V_{2}(I(n+1))-V_{2}(I(n))$, we also

$$
\begin{aligned}
& \Delta V_{2}(n) \leq I(n+1)-I(n)-\frac{G\left(I^{*}\right)}{G(n+1)} \\
& \times(I(n+1)-I(n)) \\
& =\left(1-\frac{G\left(I^{*}\right)}{G(I(n+1))}\right) \\
& \times(F(S(n+1)) G(I(n+1)) \\
& -(k+\mu+\alpha) I(n+1)) \\
& =(k+\mu+\alpha) I^{*}\left(1-\frac{G\left(I^{*}\right)}{G(I(n+1))}\right) \\
& \times\left(\frac{F(S(n+1)) G(I(n+1))}{F\left(S^{*}\right) G\left(I^{*}\right)}-\frac{I(n+1)}{I^{*}}\right) \\
& =(k+\mu+\alpha) I^{*} \\
& \times\left(\frac{F(S(n+1)) G(I(n+1))}{F\left(S^{*}\right) G\left(I^{*}\right)}\right. \\
& -\frac{F(S(n+1))}{F\left(S^{*}\right)}-\frac{I(n+1)}{I^{*}} \\
& \left.+\frac{I(n+1)}{I^{*}} \frac{G\left(I^{*}\right)}{G(I(n+1))}\right) .
\end{aligned}
$$

Further, by computing $\Delta V_{3}(n)=V_{3}(R(n+1))-V_{3}(R(n))$, we have

$$
\begin{aligned}
& \Delta V_{3}(n)=\frac{1}{2}\left((R(n+1)-R(n))^{2}\right. \\
& +2(R(n+1)-R(n))\left(R(n)-R^{*}\right) \\
& \left.+\left(R(n)-R^{*}\right)^{2}\right) \\
& -\frac{1}{2}\left(R(n)-R^{*}\right)^{2} \\
& =\frac{1}{2}(R(n+1)-R(n))\left(R(n+1)+R(n)-2 R^{*}\right) \\
& =(R(n+1)-R(n))\left(R(n+1)-R^{*}\right) \\
& -\frac{1}{2}(R(n+1)-R(n))^{2} \\
& \leq\left(R(n+1)-R^{*}\right)(R(n+1)-R(n)) \\
& =\left(R(n+1)-R^{*}\right)(k I(n+1)-(\mu+\gamma) R(n+1)) \\
& =\left(R(n+1)-R^{*}\right) \\
& \times\left(k\left(N(n+1)-N^{*}\right)\right. \\
& -k\left(S(n+1)-S^{*}\right) \\
& \left.-(k+\mu+\gamma)\left(R(n+1)-R^{*}\right)\right) \\
& =k\left(R(n+1)-R^{*}\right)\left(N(n+1)-N^{*}\right) \\
& -k\left(R(n+1)-R^{*}\right) \\
& \times\left(S(n+1)-S^{*}\right) \\
& -(k+\mu+\gamma)\left(R(n+1)-R^{*}\right)^{2} .
\end{aligned}
$$

Finally, by computing $\Delta V_{4}(n)=V_{4}(N(n+1), R(n+1))-$ $V_{4}(N(n), R(n))$, we further have

$$
\begin{aligned}
\Delta V_{4}(n)=\frac{1}{2}[ & \left(N(n+1)-N(n)+\frac{\alpha}{k}(R(n+1)-R(n))\right)^{2} \\
& +2\left(N(n+1)-N(n)+\frac{\alpha}{k}(R(n+1)-R(n))\right) \\
& \left.\times\left(N(n)-N^{*}+\frac{\alpha}{k}\left(R(n)-R^{*}\right)\right)\right] \\
=\frac{1}{2}( & \left.N(n+1)-N(n)+\frac{\alpha}{k}(R(n+1)-R(n))\right) \\
\times & \left(N(n+1)+N(n)+\frac{\alpha}{k}(R(n+1)+R(n))\right. \\
& \left.-2\left(N^{*}-\frac{\alpha}{k} R^{*}\right)\right)
\end{aligned}
$$




$$
\begin{aligned}
& =\left(N(n+1)-N(n)+\frac{\alpha}{k}(R(n+1)-R(n))\right) \quad \text { we have } \\
& \times\left(N(n+1)-N^{*}+\frac{\alpha}{k}\left(R(n+1)-R^{*}\right)\right) \quad \Delta V(n) \leq-\mu \omega_{2}\left(N(n+1)-N^{*}\right)^{2} \\
& -\frac{1}{2}\left(N(n+1)-N^{*}+\frac{\alpha}{k}\left(R(n+1)-R^{*}\right)\right)^{2} \quad-\left(\frac{\omega_{2} \alpha^{2}}{k^{2}}(\mu+\gamma)+\omega_{1}(k+\mu+\gamma)\right) \\
& \leq\left(\left(N(n+1)-N^{*}\right)+\frac{\alpha}{k}\left(R(n+1)-R^{*}\right)\right) \quad \times\left(R(n+1)-R^{*}\right)^{2} \\
& \times\left((N(n+1)-N(n))+\frac{\alpha}{k}(R(n+1)-R(n))\right) \quad-\mu\left(1-\frac{F\left(S^{*}\right)}{F(S(n+1))}\right)\left(S(n+1)-S^{*}\right) \\
& =\left(\left(N(n+1)-N^{*}\right)+\frac{\alpha}{k}\left(R(n+1)-R^{*}\right)\right)+\gamma\left(1-\frac{F\left(S^{*}\right)}{F(S(n+1))}\right)\left(R(n+1)-R^{*}\right) \\
& \times\left((\Lambda-\mu N(n+1)-\alpha I(n+1)) \quad-\omega_{1} k\left(R(n+1)-R^{*}\right)\left(S(n+1)-S^{*}\right)\right. \\
& \left.+\frac{\alpha}{k}(k I(n+1)-(\mu+\gamma) R(n+1))\right) \quad+(k+\mu+\alpha) I^{*} \\
& =\left(\left(N(n+1)-N^{*}\right)+\frac{\alpha}{k}\left(R(n+1)-R^{*}\right)\right) \\
& \times\left(\mu N^{*}-\mu N(n+1)\right. \\
& \left.-\frac{\alpha(\mu+\gamma)}{k}\left(R(n+1)-R^{*}\right)\right) \\
& =-\mu\left(N(n+1)-N^{*}\right)^{2}-\frac{\alpha^{2}}{k^{2}}(\mu+\gamma) \\
& \times\left(R(n+1)-R^{*}\right)^{2}
\end{aligned}
$$$$
-\frac{\alpha(2 \mu+\gamma)}{k}\left(N(n+1)-N^{*}\right)\left(R(n+1)-R^{*}\right) .
$$

Now, we define a Lyapunov function as follows:

$$
\omega_{1}=\frac{\gamma F\left(S^{*}\right)}{k\left(S^{*}\right)^{2}}, \quad \omega_{2}=\frac{k \gamma F\left(S^{*}\right)}{\alpha(2 \mu+\gamma)\left(S^{*}\right)^{2}} .
$$

$V(S, I, R)=V_{1}(S)+V_{2}(I)+\omega_{1} V_{3}(R)+\omega_{2} V_{4}(N, R)$,

where $\omega_{1}$ and $\omega_{2}$ are positive constants which will be chosen in the following. It is obvious that from (40) and (41) $V(S, I, R)>0$ for all $(S, I, R) \neq\left(S^{*}, I^{*}, R^{*}\right)$ and $V(S, I, R)=0$ if and only if $(S, I, R)=\left(S^{*}, I^{*}, R^{*}\right)$. By computing

$$
\begin{aligned}
\Delta V(n)= & V(S(n+1), I(n+1), R(n+1)) \\
& -V(S(n),(n), R(n))
\end{aligned}
$$

Choose constants $\omega_{1}$ and $\omega_{2}$ as follows:

hen we further have

$$
\begin{aligned}
\Delta V(n) \leq & -\frac{\mu k^{2} \gamma F\left(S^{*}\right)}{\alpha(2 \mu+\gamma)\left(S^{*}\right)^{2}}\left(N(n+1)-N^{*}\right)^{2} \\
& -\frac{\gamma F\left(S^{*}\right)}{\left(S^{*}\right)^{2}}\left(\frac{\alpha(\mu+\gamma)}{k(2 \mu+\gamma)}+\frac{\mu+\gamma}{k}+1\right) \\
& \times\left(R(n+1)-R^{*}\right)^{2} \\
& +\frac{F\left(S^{*}\right)}{\left(S^{*}\right)^{2}}\left(S(n+1)-S^{*}\right)\left(1-\frac{F\left(S^{*}\right)}{F(S(n+1))}\right)
\end{aligned}
$$




$$
\begin{aligned}
& \times\left(-\frac{\mu\left(S^{*}\right)^{2}}{F\left(S^{*}\right)}+\frac{\gamma\left(S^{*}\right)^{2}}{F\left(S^{*}\right)} \frac{R(n+1)-R^{*}}{S(n+1)-S^{*}}\right. \\
& \left.-\gamma\left(R(n+1)-R^{*}\right) \frac{F(S(n+1))}{F(S(n+1))-F\left(S^{*}\right)}\right) \\
& +(k+\mu+\alpha) I^{*} \\
& \times\left(\left(2-\frac{F\left(S^{*}\right)}{F(S(n+1))}-\frac{F(S(n+1))}{F\left(S^{*}\right)}\right)\right. \\
& +\left(\frac{G\left(I^{*}\right)}{G(I(n+1))}-1\right) \\
& \left.\times\left(\frac{I(n+1)}{I^{*}}-\frac{G(I(n+1))}{G\left(I^{*}\right)}\right)\right) \\
& =-\frac{\mu k^{2} \gamma F\left(S^{*}\right)}{\alpha(2 \mu+\gamma)\left(S^{*}\right)^{2}}\left(N(n+1)-N^{*}\right)^{2} \\
& -\frac{\gamma F\left(S^{*}\right)}{\left(S^{*}\right)^{2}}\left(\frac{\alpha(\mu+\gamma)}{k(2 \mu+\gamma)}+\frac{\mu+\gamma}{k}+1\right) \\
& \times\left(R(n+1)-R^{*}\right)^{2} \\
& +\frac{F\left(S^{*}\right)}{\left(S^{*}\right)^{2}}\left(S(n+1)-S^{*}\right)\left(1-\frac{F\left(S^{*}\right)}{F(S(n+1))}\right) \\
& \times\left(-\frac{\mu F\left(S^{*}\right)}{\left(S^{*}\right)^{2}}-\gamma\left(R(n+1)-R^{*}\right)\right. \\
& \times\left(\frac{F(S(n+1))}{F(S(n+1))-F\left(S^{*}\right)}\right. \\
& \left.\left.-\frac{\left(S^{*}\right)^{2}}{F\left(S^{*}\right)\left(S(n+1)-S^{*}\right)}\right)\right) \\
& +(k+\mu+\alpha) I^{*} \\
& \times\left(\left(2-\frac{F\left(S^{*}\right)}{F(S(n+1))}-\frac{F(S(n+1))}{F\left(S^{*}\right)}\right)\right. \\
& +\left(\frac{G\left(I^{*}\right)}{G(I(n+1))}-1\right) \\
& \left.\times\left(\frac{I(n+1)}{I^{*}}-\frac{G(I(n+1))}{G\left(I^{*}\right)}\right)\right) .
\end{aligned}
$$

Noting that $F(S)>0$, for all $S>0$, then we have

$$
2-\frac{F\left(S^{*}\right)}{F(S(n+1))}-\frac{F(S(n+1))}{F\left(S^{*}\right)} \leq 0 .
$$

From $\left(H_{1}\right)$, it follows that $F(S(n+1)) \geq F\left(S^{*}\right)$ when $S(n+1) \geq$ $S^{*}$ and $F(S(n+1)) \leq F\left(S^{*}\right)$ when $S(n+1) \leq S^{*}$. Hence, we have the following inequality:

$$
\left(S(n+1)-S^{*}\right)\left(1-\frac{F\left(S^{*}\right)}{F(S(n+1))}\right) \geq 0 .
$$

Furthermore, from $\left(H_{1}\right)$, we also have the following inequalities:

$$
\begin{aligned}
& \frac{G(I(n+1))}{G\left(I^{*}\right)} \geq \frac{I(n+1)}{I^{*}} \quad \text { if } 0<I(n+1) \leq I^{*}, \\
& \frac{G(I(n+1))}{G\left(I^{*}\right)} \leq \frac{I(n+1)}{I^{*}} \quad \text { if } I(n+1) \geq I^{*},
\end{aligned}
$$

which implies that

$$
\left(\frac{G\left(I^{*}\right)}{G(I(n+1))}-1\right)\left(\frac{I(n+1)}{I^{*}}-\frac{G(I(n+1))}{G\left(I^{*}\right)}\right) \leq 0 .
$$

From (53), (54), and (56), we further obtain

$$
\begin{aligned}
\Delta V(n) \leq & -\frac{\mu k^{2} \gamma F\left(S^{*}\right)}{\alpha(2 \mu+\gamma)\left(S^{*}\right)^{2}}\left(N(n+1)-N^{*}\right)^{2} \\
& -\frac{\gamma F\left(S^{*}\right)}{\left(S^{*}\right)^{2}}\left(\frac{\alpha(\mu+\gamma)}{k(2 \mu+\gamma)}+\frac{\mu+\gamma}{k}+1\right) \\
& \times\left(R(n+1)-R^{*}\right)^{2} \\
+ & \frac{F\left(S^{*}\right)}{\left(S^{*}\right)^{2}}\left(S(n+1)-S^{*}\right)\left(1-\frac{F\left(S^{*}\right)}{F(S(n+1))}\right) \\
\times & {\left[\begin{array}{l}
-\frac{\mu F\left(S^{*}\right)}{\left(S^{*}\right)^{2}}+\gamma\left(R^{*}-R(n+1)\right) \\
\times\left(\frac{F(S(n+1))}{F(S(n+1))-F\left(S^{*}\right)}\right. \\
\end{array}\right.} \\
& \left.\left.-\frac{\left(S^{*}\right)^{2}}{F\left(S^{*}\right)\left(S(n+1)-S^{*}\right)}\right)\right] .
\end{aligned}
$$

From $\left(\mathrm{H}_{2}\right)$ and $\left(\mathrm{H}_{3}\right)$, we finally have $\Delta V(n) \leq 0$ for all $n \geq 0$. Obviously, $\Delta V(n)=0$ if and only if $S(n)=S^{*}, I(n)=I^{*}$, and $R(n)=R^{*}$ for all $n \geq 0$. Therefore, using the theorems of stability of the difference equations (see Theorem 6.3 in [33]), we obtain that $E^{*}$ is globally asymptotically stable. This completes the proof.

As a special case of model (1), we consider the rate of losing immunity $\gamma=0$ in model (1); that is, model (1) degenerates into a SIR epidemic model. Then, in the above 
calculation of $\Delta V(n)$, we can directly obtain the following inequality without $\left(H_{2}\right)$ and $\left(H_{3}\right)$ :

$$
\begin{aligned}
\Delta V(n) \leq & -\mu\left(S(n+1)-S^{*}\right)\left(1-\frac{F\left(S^{*}\right)}{F(S(n+1))}\right) \\
+ & (k+\mu+\alpha) I^{*} \\
\times & \left(\left(2-\frac{F\left(S^{*}\right)}{F(S(n+1))}-\frac{F(S(n+1))}{F\left(S^{*}\right)}\right)\right. \\
& +\left(\frac{G\left(I^{*}\right)}{G(I(n+1))}-1\right) \\
& \left.\times\left(\frac{I(n+1)}{I^{*}}-\frac{G(I(n+1))}{G\left(I^{*}\right)}\right)\right) .
\end{aligned}
$$

We have $\Delta V(n) \leq 0$ for all $n \geq 0$ and $\Delta V(n)=0$ if and only if $S(n)=S^{*}$ and $I(n)=I^{*}$ for all $n \geq 0$. Therefore, as a consequence of Theorem 5, we have the following result.

Corollary 6. Assume that $\left(H_{1}\right)$ holds and $\gamma=0$ in model (1). Then endemic equilibrium $E^{*}\left(S^{*}, I^{*}, R^{*}\right)$ is globally asymptotically stable if and only if $\mathscr{R}_{0}>1$.

Remark 7. By comparing the results obtained in [24], then, from Corollary 6, we see that Theorem 5 is a direct extension of the corresponding result given in [24] on the global stability of the endemic equilibrium in the nondelayed case and the recovered individuals are in a position to lose the immunity.

Remark 8. For general model (1), we spontaneously expect that as long as basic reproduction number $\mathscr{R}_{0}>1$, then model (1) shows a unique endemic equilibrium which is globally asymptotically stable. However, it is a pity that, in Theorem 5 , in order to obtain the global asymptotic stability of endemic equilibrium $E^{*}$, we need to introduce some additional conditions, that is, $\left(H_{2}\right)$ and $\left(H_{3}\right)$. Furthermore, from the proof of Theorem 5 , we easily see that assumptions $\left(\mathrm{H}_{2}\right)$ and $\left(\mathrm{H}_{3}\right)$ only are used to ensure $\Delta V(n) \leq 0$ for all $n \geq 0$. Therefore, an important open problem is whether we can directly prove $\Delta V(n) \leq 0$ for all $n \geq 0$ without assumptions $\left(\mathrm{H}_{2}\right)$ and $\left(\mathrm{H}_{3}\right)$ and further obtain the global asymptotic stability of endemic equilibrium $E^{*}$ of model (1) only when basic reproduction number $\mathscr{R}_{0}>1$.

\section{Permanence of Disease}

In this section, we will use the theory of persistence in general discrete dynamical systems to study the permanence of model (1). We will obtain that the disease in model (1) is permanent only when basic reproduction number $\mathscr{R}_{0}>1$ and assumption $\left(H_{1}\right)$ holds.

Let $X$ be a metric space with metric $d$ and let $f: X \rightarrow$ $X$ be a continuous map. For any $x_{0} \in X$, the sequence $\left\{x_{n}\right\}$ defined by $x_{n+1}=f\left(x_{n}\right)$ for any integer $n \geq 0$ is said to be a solution sequence through $x_{0}$, and the omega limit set of $\left\{x_{n}\right\}$ is defined by $\omega\left(x_{0}\right)=\left\{y \in X\right.$ : there is a sequence $n_{k} \rightarrow \infty$ such that $\left.\lim _{k \rightarrow \infty} x_{n_{k}}=y\right\}$. For a nonempty set $M \subset X$, we further define the stable set of $M$ by $W^{s}(M)=\left\{x_{0} \in X\right.$ : $\left.\lim _{n \rightarrow \infty} d\left(x_{n}, M\right)=0\right\}$.

Let $X_{0}$ be a nonempty open set of $X$. We denote

$$
\partial X_{0}:=X \backslash X_{0}, \quad M_{\partial}:=\left\{x_{0} \in \partial X_{0}: x_{n} \in \partial X_{0} \forall n \geq 0\right\} .
$$

Lemma 9. Let $f: X \rightarrow X$ be a continuous map. Assume that the following conditions hold.

$\left(C_{1}\right) f$ is compact and point dissipative, and $f\left(X_{0}\right) \subseteq X_{0}$.

$\left(C_{2}\right)$ There exists a finite sequence $\mathscr{M}=\left\{M_{1}, \ldots, M_{k}\right\}$ of compact and isolated invariant sets such that

(a) $M_{i} \cap M_{j}=\emptyset$ for any $i, j=1,2, \ldots, k$ and $i \neq j$;

(b) $\Omega\left(M_{\partial}\right):=\bigcup_{x \in M_{\partial}} \omega(x) \subset \bigcup_{i=1}^{k} M_{i}$;

(c) no subset of $\mathscr{M}$ forms a cycle in $\partial X_{0}$;

(d) $W^{s}\left(M_{i}\right) \cap X_{0}=\emptyset$ for each $1 \leq i \leq k$.

Then $f$ is uniformly persistent with respect to $\left.\left(X_{0}, \partial X_{0}\right)\right)$; that is, there exists a constant $\eta>0$ such that $\liminf _{n \rightarrow \infty} d\left(x_{n}, \partial X_{0}\right) \geq \eta$ for all $x_{0} \in X_{0}$.

Here, the definitions on the compactness and point dissipativity of map $f$ and the definitions on the compactness, isolated invariance, and the cycle in $\partial X_{0}$ for sequence $\mathscr{M}=$ $\left\{M_{1}, \ldots, M_{k}\right\}$ can be found in [34]. Furthermore, Lemma 9 can be obtained from Theorem 1.1.3, Theorem 1.3.1, Remark 1.3.1, and Theorem 1.3.3 given by Zhao in [34].

On the permanence of the disease for model (1), we have the following result.

Theorem 10. Assume that $\left(H_{1}\right)$ holds. Then, the disease in model (1) is permanent; that is, there are two constants $M>$ $m>0$ such that

$$
m \leq \liminf _{n \rightarrow \infty} I(n) \leq \limsup _{n \rightarrow \infty} I(n) \leq M,
$$

for any positive solution $(S(n), I(n), R(n))$ of model (1) if and only if $\mathscr{R}_{0}>1$.

Proof. From Theorem 4 we see that the necessity is obvious. Now, we only need to prove the sufficiency. Define two sets as follows:

$$
\begin{aligned}
& X=\left\{(S, I, R) \in R^{3}: S>0, I \geq 0, R \geq 0\right\}, \\
& X_{0}=\{(S, I, R) \in X: S>0, I>0, R \geq 0\} .
\end{aligned}
$$

We have

$$
\partial X_{0}=X \backslash X_{0}=\{(S, I, R): S>0, I=0, R \geq 0\} .
$$

For any initial point $x_{0}=\left(S_{0}, I_{0}, R_{0}\right) \in X$, let $x_{n}=$ $(S(n), I(n), R(n))$ be the solution of model (1) through $x_{0}$. We define map $f: X \rightarrow X$ by $f\left(x_{0}\right)=x_{1}$.

From the positivity and ultimate boundedness of solutions of model (1), we obtain $f\left(X_{0}\right) \subseteq X_{0}$ and $f$ is also point dissipative. 
By observing the proof of Theorem 3, we see that, since $N(1)$ and $R(1)$ are continuous with respect to $N(0)$ and $R(0)$, respectively, $\Psi(y)$ is also continuous with respect to $x_{0}=$ $\left(S_{0}, I_{0}, R_{0}\right)$. Hence, $I(1)$, as the solution of $\Psi(y)=0$, is also continuous for $x_{0}$. Similarly, from the expression of $\Phi(x)$ and the continuity of $I(1)$ with respect to $x_{0}$, we obtain that $\Phi(x)$ is continuous with respect to $x_{0}$. Hence, $S(1)$, as the solution of $\Phi(x)=0$, is also continuous for $x_{0}$. Therefore, we finally obtain that map $f$ is continuous on $X$. From this, we obtain that $f$ also is compact.

In $\partial X_{0}$, we have $I(n) \equiv 0$, and hence $(S(n), R(n))$ satisfies

$$
\begin{aligned}
& S(n+1)=S(n)+\Lambda-\mu S(n+1)+\gamma R(n+1), \\
& R(n+1)=R(n)-(\mu+\gamma) R(n+1) .
\end{aligned}
$$

Obviously, we can obtain $(S(n), R(n)) \rightarrow(\Lambda / \mu, 0)$ as $n \rightarrow$ $\infty$. This shows that $\omega\left(x_{0}\right)=\left\{E^{0}\right\}$ for any $x_{0} \in M_{\partial}$ and $\Omega\left(M_{\partial}\right)=\bigcup_{x \in M_{\partial}} \omega(x)=\left\{E^{*}\right\}$. Choose $\mathscr{M}=\left\{E^{0}\right\}$; then we easily see that conditions (a)-(c) of Lemma 9 hold.

Now, we prove that condition (d) in Lemma 9 also holds. Otherwise, there is a point $\left(S_{0}, I_{0}, R_{0}\right) \in X_{0}$ such that $(S(n), I(n), R(n)) \rightarrow E^{0}$ as $n \rightarrow \infty$. From $\mathscr{R}_{0}>1$, we can choose a small enough constant $\varepsilon>0$ such that

$$
\left(F\left(\frac{\Lambda}{\mu}\right)-\varepsilon\right)\left(G^{\prime}(0)-\varepsilon\right)-(k+\mu+\alpha)>0 .
$$

Since $\lim _{n \rightarrow \infty} S(n)=\Lambda / \mu$ and $\lim _{n \rightarrow \infty}(G(I(n)) / I(n))=$ $G^{\prime}(0)$, there exists $N>0$ such that $F(S(n+1))>F(\Lambda / \mu)-\varepsilon$ and $G(I(n+1)) / I(n+1)>G^{\prime}(0)-\varepsilon$ for all $n>N$. Therefore, we have

$$
\begin{aligned}
& I(n+1) \\
& =I(n)+F(S(n+1)) G(I(n+1))-(k+\mu+\alpha) I(n+1) \\
& \geq I(n)+\left[\left(F\left(\frac{\Lambda}{\mu}\right)-\varepsilon\right)\left(G^{\prime}(0)-\varepsilon\right)-(k+\mu+\alpha)\right] \\
& \quad \times I(n+1),
\end{aligned}
$$

for all $n>N$. Consequently,

$$
\begin{aligned}
& I(n+1)\left[1+k+\mu+\alpha-\left(F\left(\frac{\Lambda}{\mu}\right)-\varepsilon\right)\left(G^{\prime}(0)-\varepsilon\right)\right] \\
& \quad \geq I(n),
\end{aligned}
$$

for all $n>N$. Since $0 \leq 1+k+\mu+\alpha-(F(\Lambda / \mu)-\varepsilon)\left(G^{\prime}(0)-\varepsilon\right)<1$, we can finally obtain from $(66)$ that $\lim _{n \rightarrow \infty} I(n)=\infty$, which leads to a contradiction. Therefore, condition (d) in Lemma 9 holds. Finally, from Lemma 9 we obtain that the disease in model (1) is permanent. This completes the proof.

Remark 11. From Theorem 10, we directly see that assumptions $\left(\mathrm{H}_{2}\right)$ and $\left(\mathrm{H}_{3}\right)$ only are used to obtain the global asymptotic stability of endemic equilibrium $E^{*}$.

\section{Special Case $F(S)=S /(1+\lambda S)$}

Now, we especially discuss the special case of model (1): $F(S)=S /(1+\lambda S)$, where $\lambda \geq 0$ is a constant. Firstly, when $F(S)=S /(1+\lambda S)$, the basic reproduction number of model (1) becomes

$$
\mathscr{R}_{0}=\frac{F(\Lambda / \mu) G^{\prime}(0)}{k+\mu+\alpha}=\frac{\Lambda G^{\prime}(0)}{(\mu+\lambda \Lambda)(k+\mu+\alpha)} .
$$

Furthermore, by calculating, we obtain that $\left(\mathrm{H}_{2}\right)$ naturally holds, and assumption $\left(H_{3}\right)$ is equivalent to the following simple form:

$$
\gamma R^{*}-\mu S^{*} \leq 0
$$

Therefore, as a direct consequence of Theorem 5, we firstly have the following corollary.

Corollary 12. Assume that $\left(H_{1}\right)$ holds and $F(S)=S /(1+\lambda S)$, where $\lambda \geq 0$ is a constant. If $\mathscr{R}_{0}>1$ and inequality (68) holds, then endemic equilibrium $E^{*}\left(S^{*}, I^{*}, R^{*}\right)$ of model (1) is globally asymptotically stable.

Furthermore, in order to validate inequality (68), we have the following result.

Theorem 13. Assume that $\left(H_{1}\right)$ holds and $F(S)=S /(1+\lambda S)$ with $\lambda \geq 0$ is a constant. Then inequality (68) holds if one of the following conditions holds:

(1) $1<\mathscr{R}_{0} \leq(\mu(k+\mu+\alpha)+\Lambda k \lambda) / k(\mu+\lambda \Lambda)$,

(2) $\mathscr{R}_{0}>(\mu(k+\mu+\alpha)+\Lambda k \lambda) / k(\mu+\lambda \Lambda)$ and $0 \leq \gamma \leq$ $\bar{\gamma}:=\mu / L$, where

$$
L=\frac{\mathscr{R}_{0}(\mu+\lambda \Lambda) k}{\mu(k+\mu+\alpha)}-\frac{\mu(k+\mu+\alpha)+\Lambda k \lambda}{\mu(k+\mu+\alpha)} .
$$

Proof. When $F(S) G(I)=(S /(1+\lambda S)) G(I)$, endemic equilibrium $E^{*}\left(S^{*}, I^{*}, R^{*}\right)$ of model (1) satisfies

$$
\begin{aligned}
& \Lambda=\frac{S^{*}}{1+\lambda S^{*}} G\left(I^{*}\right)+\mu S^{*}-\gamma R^{*}, \\
& I^{*}(k+\mu+\alpha)=\frac{S^{*}}{1+\lambda S^{*}} G\left(I^{*}\right), \\
& k I^{*}=(\mu+\gamma) R^{*} .
\end{aligned}
$$

From the second and third equations of (70), we obtain

$$
S^{*}=\frac{I^{*}(k+\mu+\alpha)}{G\left(I^{*}\right)-\lambda(k+\mu+\alpha) I^{*}}, \quad R^{*}=\frac{k I^{*}}{\mu+\gamma} .
$$

Putting (71) into the first equation of (70), we have

$$
\begin{aligned}
I^{*}= & \left(\Lambda-\frac{\mu(k+\mu+\alpha) I^{*}}{G\left(I^{*}\right)-\lambda(k+\mu+\alpha) I^{*}}\right) \\
& \times \frac{\mu+\gamma}{\mu(k+\mu+\alpha)+\gamma(\mu+\alpha)} .
\end{aligned}
$$


Hence, from (71) and (72), we obtain

$$
\begin{aligned}
& \mu S^{*}-\gamma R^{*}=\frac{\mu(k+\mu+\alpha) I^{*}}{G\left(I^{*}\right)-\lambda(k+\mu+\alpha) I^{*}}-\gamma R^{*} \\
& =\frac{\mu(k+\mu+\alpha)(\mu+\gamma) R^{*}}{\left(G\left(I^{*}\right)-\lambda(k+\mu+\alpha) I^{*}\right) k}-\gamma R^{*} \\
& =\frac{R^{*}}{k\left(G\left(I^{*}\right)-\lambda(k+\mu+\alpha) I^{*}\right)} \\
& \times[\mu(k+\mu+\alpha)(\mu+\gamma) \\
& \left.-k \gamma\left(\frac{G\left(I^{*}\right)}{I^{*}}-\lambda(k+\mu+\alpha)\right) I^{*}\right] \\
& =\frac{R^{*}}{k\left(G\left(I^{*}\right)-\lambda(k+\mu+\alpha) I^{*}\right)} \\
& \times[\mu(k+\mu+\alpha)(\mu+\gamma) \\
& -k \gamma\left(\frac{G\left(I^{*}\right)}{I^{*}}-\lambda(k+\mu+\alpha)\right) \\
& \times\left(\Lambda-\frac{\mu(k+\mu+\alpha) I^{*}}{G\left(I^{*}\right)-\lambda(k+\mu+\alpha) I^{*}}\right) \\
& \left.\times \frac{\mu+\gamma}{\mu(k+\mu+\alpha)+\gamma(\mu+\alpha)}\right] \\
& =\frac{R^{*}}{k\left(G\left(I^{*}\right)-\lambda(k+\mu+\alpha) I^{*}\right)} \\
& \times[\mu(k+\mu+\alpha)(\mu+\gamma) \\
& -\frac{\mu+\gamma}{\mu(k+\mu+\alpha)+\gamma(\mu+\alpha)} \\
& \times\left(\Lambda \gamma k\left(\frac{G\left(I^{*}\right)}{I^{*}}-\lambda(k+\mu+\alpha)\right)\right. \\
& -k \gamma \mu(k+\mu+\alpha))]
\end{aligned}
$$

Since $G(I) / I$ is nonincreasing for all $I>0$ in $\left(H_{1}\right)$, we have

$$
\frac{G\left(I^{*}\right)}{I^{*}}-\lambda(k+\mu+\alpha) \leq G^{\prime}(0)-\lambda(k+\mu+\alpha) .
$$

Therefore, from (73), we further have

$$
\begin{aligned}
& \mu S^{*}-\gamma R^{*} \geq \frac{R^{*}}{k\left(G\left(I^{*}\right)-\lambda(k+\mu+\alpha) I^{*}\right)} \\
& \times(\mu(k+\mu+\alpha)(\mu+\gamma) \\
& -\frac{\mu+\gamma}{\mu(k+\mu+\alpha)+\gamma(\mu+\alpha)} \\
& \times\left(\Lambda \gamma k\left(G^{\prime}(0)-\lambda(k+\mu+\alpha)\right)\right. \\
& -k \gamma \mu(k+\mu+\alpha))) \\
& =\frac{R^{*}}{k\left(G^{\prime}\left(I^{*}\right)-\lambda(k+\mu+\alpha) I^{*}\right)} \\
& \times \frac{\mu(\mu+\gamma)(k+\mu+\alpha)^{2}}{\mu(k+\mu+\alpha)+\gamma(\mu+\alpha)} \\
& \times\left[\frac{1}{k+\mu+\alpha}(\mu(k+\mu+\alpha)+\gamma(\mu+\alpha))\right. \\
& +\frac{k \gamma}{k+\mu+\alpha} \\
& \left.-\frac{\Lambda k \gamma\left(G^{\prime}(0)-\lambda(k+\mu+\alpha)\right)}{\mu(k+\mu+\alpha)^{2}}\right] \\
& =\frac{R^{*}}{k\left(G^{\prime}\left(I^{*}\right)-\lambda(k+\mu+\alpha) I^{*}\right)} \\
& \times \frac{\mu(\mu+\gamma)(k+\mu+\alpha)^{2}}{\mu(k+\mu+\alpha)+\gamma(\mu+\alpha)} \\
& \times\left(\mu+\gamma \frac{\mu(\mu+k+\alpha)+\Lambda k \lambda}{\mu(k+\mu+\alpha)}\right. \\
& \left.-\frac{\Lambda k \lambda G^{\prime}(0)}{\mu(k+\mu+\alpha)^{2}}\right) \\
& =\frac{R^{*}}{k\left(G^{\prime}\left(I^{*}\right)-\lambda(k+\mu+\alpha) I^{*}\right)} \\
& \times \frac{\mu(\mu+\gamma)(k+\mu+\alpha)^{2}}{\mu(k+\mu+\alpha)+\gamma(\mu+\alpha)} \\
& \times\left(\mu-\gamma\left(\frac{\mathscr{R}_{0}(\mu+\lambda \Lambda) k}{\mu(k+\mu+\alpha)}\right.\right. \\
& \left.\left.-\frac{\mu(\mu+k+\alpha)+\Lambda k \lambda}{\mu(k+\mu+\alpha)}\right)\right) .
\end{aligned}
$$

From (75), we obtain that, when the conditions of Theorem 10 hold, $\mu S^{*}-\gamma R^{*} \geq 0$. Therefore, inequality (68) holds. This completesthe proof. 
Remark 14. Obviously, from the above discussion for special case $F(S)=S /(1+\lambda S)$ of model (1), we also have an important open problem, that is, whether endemic equilibrium $E^{*}$ of model (1) is globally asymptotically stable as long as basic reproduction number $\mathscr{R}_{0}>1$.

In the following, we will give an affirmative answer for above open problem in allusion to $F(S)=S$ and $G(I)=$ $\beta I /(1+\omega I)$ in model (1), by constructing the other Lyapunov function which is different from the Lyapunov function used in Theorem 5.

Firstly, we see in model (1) when $F(S)=S$ and $G(I)=$ $\beta I /(1+\omega I)$, where $\beta>0$ and $\omega \geq 0$ are two constants, basic reproduction number

$$
\mathscr{R}_{0}=\frac{F(\Lambda / \mu) G^{\prime}(0)}{k+\mu+\alpha}=\frac{\Lambda \beta}{\mu(k+\mu+\alpha)},
$$

and assumption $\left(H_{1}\right)$ naturally holds. Therefore, from Theorem 2, when $\mathscr{R}_{0}>1$, model (1) has a unique endemic equilibrium $E^{*}\left(S^{*}, I^{*}, R^{*}\right)$.

Theorem 15. When $F(S) G(I)=\beta S I /(1+\omega I)$ in model (1), then endemic equilibrium $E^{*}$ is globally asymptotically stable if $\mathscr{R}_{0}>1$.

Proof. We consider the following Lyapunov function:

$$
\begin{aligned}
U(S, I, R)= & \frac{\beta}{2 \alpha}\left(N-N^{*}\right)^{2}+\left(I-I^{*}-I^{*} \ln \frac{I}{I^{*}}\right) \\
& +\frac{\omega}{2}\left(I-I^{*}\right)^{2}+\frac{\beta}{2 k}\left(R-R^{*}\right)^{2} .
\end{aligned}
$$

It is clear that $U(S, I, R)>0$ for all $(S, I, R) \neq\left(S^{*}, I^{*}, R^{*}\right)$ and $U(S, I, R)=0$ if and only if $(S, I, R)=\left(S^{*}, I^{*}, R^{*}\right)$.

Let $(S(n), I(n), R(n))$ be any positive solution of model (1). By computing

$$
\begin{aligned}
\Delta U(n)= & U(S(n+1), I(n+1), R(n+1)) \\
& -U(S(n), I(n), R(n)),
\end{aligned}
$$

a similar argument as in calculation $\Delta V_{3}(n)$ in Theorem 5 , we obtain

$$
\begin{aligned}
\Delta U(n) \leq & \frac{\beta}{\alpha}\left(N(n+1)-N^{*}\right)(N(n+1)-N(n)) \\
& +\left(I(n+1)-I(n)-I^{*} \ln \frac{I(n+1)}{I(n)}\right) \\
& +\lambda\left(I(n+1)-I^{*}\right)(I(n+1)-I(n)) \\
& +\frac{\beta}{k}\left(R(n+1)-R^{*}\right)(R(n+1)-R(n)) .
\end{aligned}
$$

By using inequality $\ln (1-x) \leq-x$ for any $x<1$, we obtain

$$
\begin{aligned}
- & \ln \frac{I(n+1)}{I(n)} \\
& =\ln 1-\left(1-\frac{I(n)}{I(n+1)}\right) \leq-\left(1-\frac{I(n)}{I(n+1)}\right) .
\end{aligned}
$$

Hence,

$$
\begin{aligned}
& \Delta U(n) \leq \frac{\beta}{\alpha}\left(N(n+1)-N^{*}\right) \\
& \times(\Lambda-\mu N-\alpha I(n+1)) \\
& +\frac{1+\omega I(n+1)}{I(n+1)} \\
& \times\left(I(n+1)-I^{*}\right)(I(n+1)-I(n)) \\
& +\frac{\beta}{k}\left(R(n+1)-R^{*}\right) \\
& \times(k I(n+1)-(\mu+\gamma) R(n+1)) \\
& =\frac{\beta}{\alpha}\left(N(n+1)-N^{*}\right)(\Lambda-\mu N-\alpha I(n+1)) \\
& +\left(I(n+1)-I^{*}\right) \\
& \times(\beta S(n+1)-(1+\omega I(n+1)) \\
& \times(k+\mu+\alpha))+\frac{\beta}{k}\left(R(n+1)-R^{*}\right) \\
& \times(k I(n+1)-(\mu+\gamma) R(n+1)) .
\end{aligned}
$$

Since $E^{*}\left(S^{*}, I^{*}, R^{*}\right)$ satisfies

$$
\begin{aligned}
& \Lambda=\mu N^{*}+\alpha I^{*}=\mu S^{*}+(\alpha+\mu) I^{*}+\mu R^{*}, \\
& k+\mu+\alpha=\frac{\beta S^{*}}{1+\omega I^{*}}, \\
& k I^{*}-(\mu+\gamma) R^{*}=0,
\end{aligned}
$$

then from (81) we further have

$$
\begin{aligned}
\Delta U(n) \leq & -\frac{\mu \beta}{\alpha}\left(N(n+1)-N^{*}\right)^{2} \\
& -\beta\left(N(n+1)-N^{*}\right)\left(I(n+1)-I^{*}\right) \\
& +\beta\left(I(n+1)-I^{*}\right) \\
& \times\left(S(n+1)-\frac{(1+\omega I(n+1)) S^{*}}{1+\omega I^{*}}\right) \\
& +\beta\left(R(n+1)-R^{*}\right)\left(I(n+1)-I^{*}\right) \\
& -\frac{\beta(\mu+\gamma)}{k}\left(R(n+1)-R^{*}\right)^{2}
\end{aligned}
$$




$$
\begin{aligned}
= & -\frac{\mu \beta}{\alpha}\left(N(n+1)-N^{*}\right)^{2} \\
& -\beta\left(N(n+1)-N^{*}\right)\left(I(n+1)-I^{*}\right) \\
& +\beta\left(I(n+1)-I^{*}\right) \\
& \times\left(\left(N(n+1)-N^{*}\right)\right. \\
& -\left(I(n+1)-I^{*}\right)-\left(R(n+1)-R^{*}\right) \\
& \left.+S^{*}-\frac{(1+\omega I(n+1)) S^{*}}{1+\omega I^{*}}\right) \\
+ & \beta\left(R(n+1)-R^{*}\right)\left(I(n+1)-I^{*}\right) \\
- & \frac{\beta(\mu+\gamma)}{k}\left(R(n+1)-R^{*}\right)^{2} \\
- & \frac{\beta(\mu+\gamma)}{k}\left(R(n+1)-R^{*}\right)^{2} \\
& \left.\frac{\left.\mu \beta(n+1)-N^{*}\right)^{2}}{\alpha}(n+1)-I^{*}\right)^{2}-\beta S^{*} \frac{\omega\left(I(n+1)-I^{*}\right)^{2}}{1+\omega I^{*}} .
\end{aligned}
$$

Therefore, we finally get that $\Delta U(n) \leq 0$ for all $n \geq 0$. Obviously, $\Delta U(n)=0$ if and only if $N(n+1)=N^{*}$, $I(n+1)=I^{*}$, and $R(n+1)=R^{*}$. Therefore, from the theorems of stability of difference equations (see Theorem 6.3 in [33]), we obtain that $E^{*}$ is globally asymptotically stable. This completes the proof.

Remark 16. By combining Theorem 4, we can obtain that, when $F(S) G(I)=\beta S I /(1+\omega I)$ in model (1), diseasefree equilibrium $E^{0}$ is globally asymptotically stable if and only if basic reproduction number $\mathscr{R}_{0} \leq 1$ and endemic equilibrium $E^{*}$ is globally asymptotically stable if and only if $\mathscr{R}_{0}>1$.

Remark 17. In [13], the author studied a continuous SIRS epidemic model with bilinear incidence rate and obtained that the disease-free equilibrium is globally stable if basic reproduction number $R_{0} \leq 1$ and the endemic equilibrium is globally stable if $R_{0}>1$. However, in this paper, we established the completely same results for the corresponding backward Euler discretization model with saturation incidence rate. This shows that the results obtained in [13] are extended and improved in the discrete models.
Remark 18. In [25], the following continuous SIRS epidemic model with a class of nonlinear incidence rates and distributed delays is considered:

$$
\begin{gathered}
\dot{S}(t)=B-\mu_{1} S(t)-\beta S(t) \int_{0}^{h} f(\tau) G(I(t-\tau)) d \tau+\delta R(t), \\
\dot{I}(t)=\beta S(t) \int_{0}^{h} f(\tau) G(I(t-\tau)) d \tau-\left(\mu_{2}+\gamma\right) I(t), \\
\dot{R}(t)=\gamma I(t)-\left(\mu_{3}+\delta\right) R(t) .
\end{gathered}
$$

By applying Lyapunov functional techniques, Enatsu et al. obtained that disease-free equilibrium $\left(B / \mu_{1}, 0,0\right)$ of model (84) is globally asymptotically stable if basic reproduction number $\mathscr{R}_{0} \leq 1$ and endemic equilibrium $\left(S^{*}, I^{*}, R^{*}\right)$ of model (84) is globally asymptotically stable if $\mathscr{R}_{0}>1$ and $\mu_{1} S^{*}-\delta R^{*} \geq 0$ hold. Comparing with the results obtained in this paper, we can see that our results are the direct extension of those in [25] for nondelayed discrete SIRS epidemic model with nonlinear incidence rate $F(S) G(I)$.

However, we also see whether the conclusions obtained in [25] can be extended to delayed discrete SIRS epidemic models with more general nonlinear incidence rate $f(S, I)$, which is left to further investigation in our future work.

\section{Numerical Simulations}

In this section, we give the following examples and numerical simulations for model (1).

Example 1. Consider

$$
F(S) G(I)=\frac{\beta S I}{1+\lambda S}
$$

We choose $\Lambda=3, \alpha=0.2, \lambda=1, \beta=0.8, \mu=0.2, \gamma=0.5$, and $k=0.3$. By calculating, we have the endemic equilibrium $\left.E^{*}=(7,3.2941,1.4118)\right)$ and the basic reproduction number

$$
\mathscr{R}_{0}=\frac{\Lambda \beta}{(k+\mu+\alpha)(\mu+\lambda \Lambda)}=1.0714>1 .
$$

However, $\gamma R^{*}-\mu S^{*}=-0.6941<0$. Clearly, inequality (56) does not hold. From the numerical simulation (see Figure 1), we obtain that the endemic equilibrium $E^{*}$ is still globally asymptotically stable. Therefore, in our future work, we expect to obtain the corresponding theoretical result for the open problem in Remark 8.

Example 2. Consider

$$
F(S) G(I)=\frac{\beta S^{2} I}{1+\lambda \sqrt{I}} .
$$

We choose $\Lambda=4, \alpha=1.2, \lambda=1, \beta=0.4, \mu=1.3$, $\gamma=0.45$, and $k=1$. By calculating, we have the endemic 


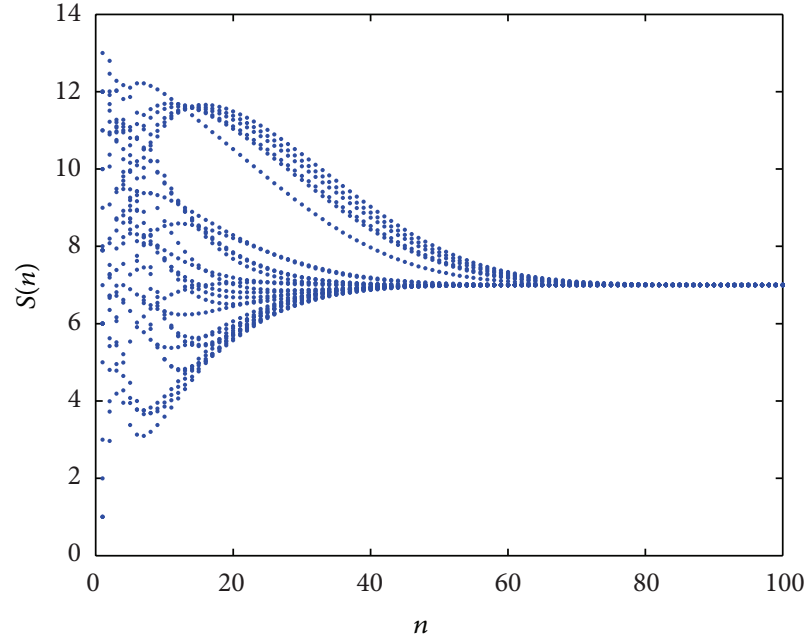

(a)

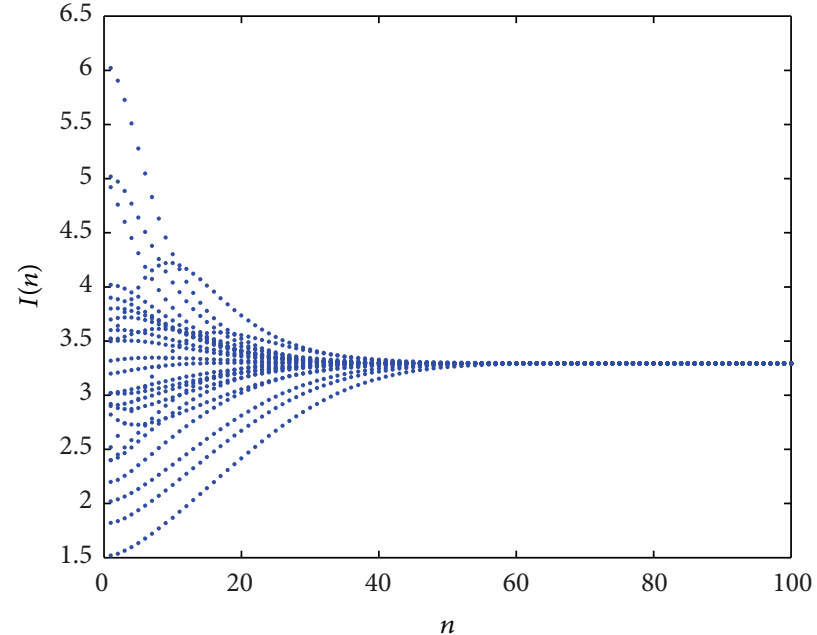

(b)

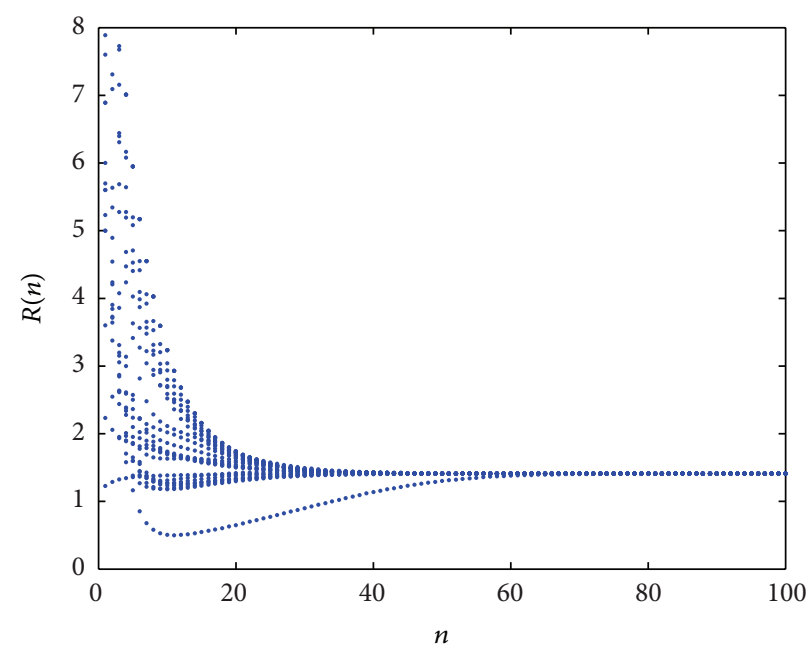

(c)

FIGURE 1: Time series of $S(n), I(n)$, and $R(n)$.

equilibrium $\left.E^{*}=(3.0637,0.0053,0.0030)\right)$ and the basic reproduction number

$$
\mathscr{R}_{0}=\frac{\Lambda^{2} \beta}{\mu^{2}(k+\mu+\alpha)}=1.082>1 .
$$

By further computation, we obtain, when $S=2.7$,

$$
\begin{aligned}
& \frac{F(S)}{F(S)-F\left(S^{*}\right)}-\frac{\left(S^{*}\right)^{2}}{F\left(S^{*}\right)\left(S-S^{*}\right)} \\
& =\frac{S^{2}}{S^{2}-\left(S^{*}\right)^{2}}-\frac{1}{S-S^{*}}=-0.7281<0,
\end{aligned}
$$

and, when $S=3.064$,

$$
\begin{aligned}
& -\frac{\mu\left(S^{*}\right)^{2}}{F\left(S^{*}\right)}+\gamma R^{*}\left(\frac{F(S)}{F(S)-F\left(S^{*}\right)}-\frac{\left(S^{*}\right)^{2}}{F\left(S^{*}\right)\left(S-S^{*}\right)}\right) \\
& =-\mu+\gamma R^{*}\left(\frac{S^{2}}{S^{2}-\left(S^{*}\right)^{2}}-\frac{1}{S-S^{*}}\right)=1.0943>0 .
\end{aligned}
$$

That is, neither $\left(\mathrm{H}_{2}\right)$ nor $\left(\mathrm{H}_{3}\right)$ holds. However, from the numerical simulation (see Figure 2), it is clear that the endemic equilibrium $E^{*}$ is still globally asymptotically stable. Therefore, in our future work, we expect to obtain the corresponding theoretical result for the open problem in Remark 8. 


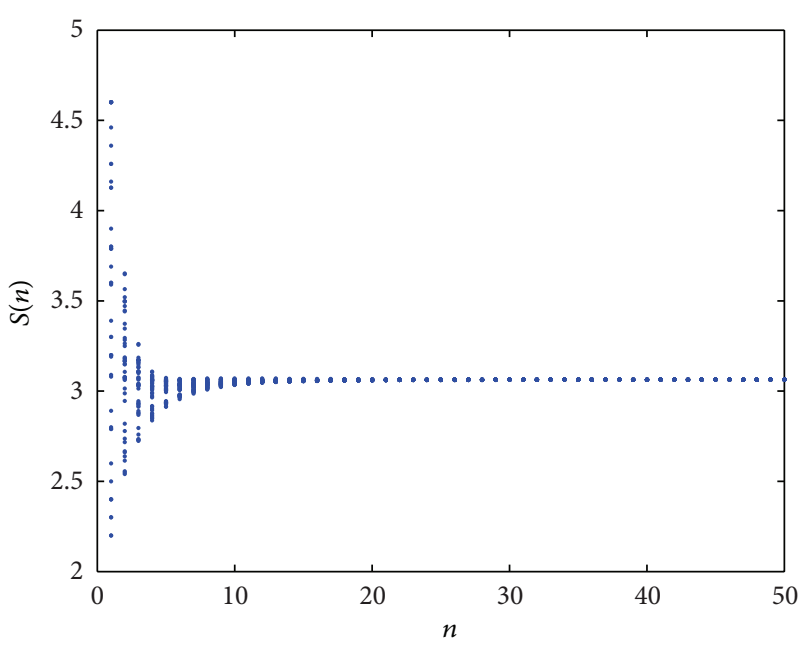

(a)

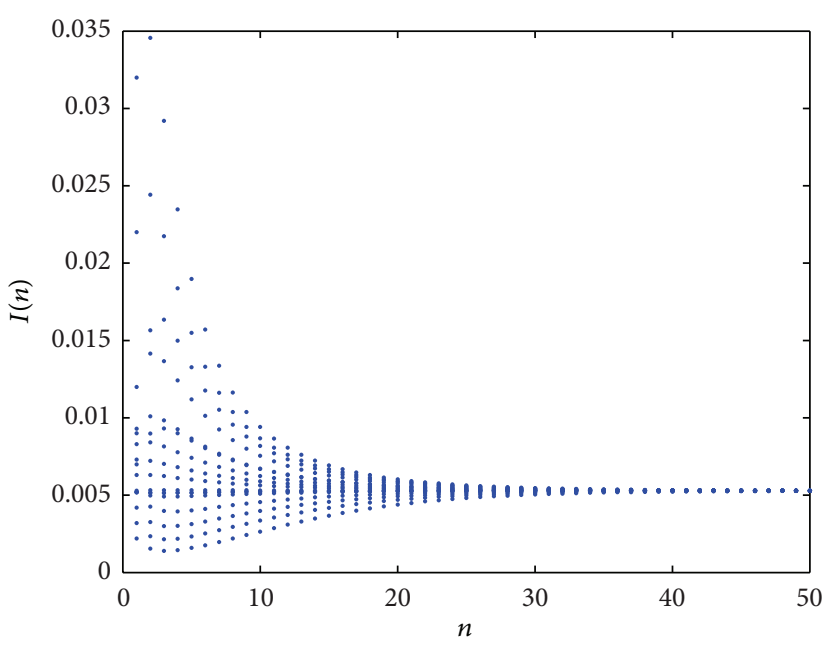

(b)

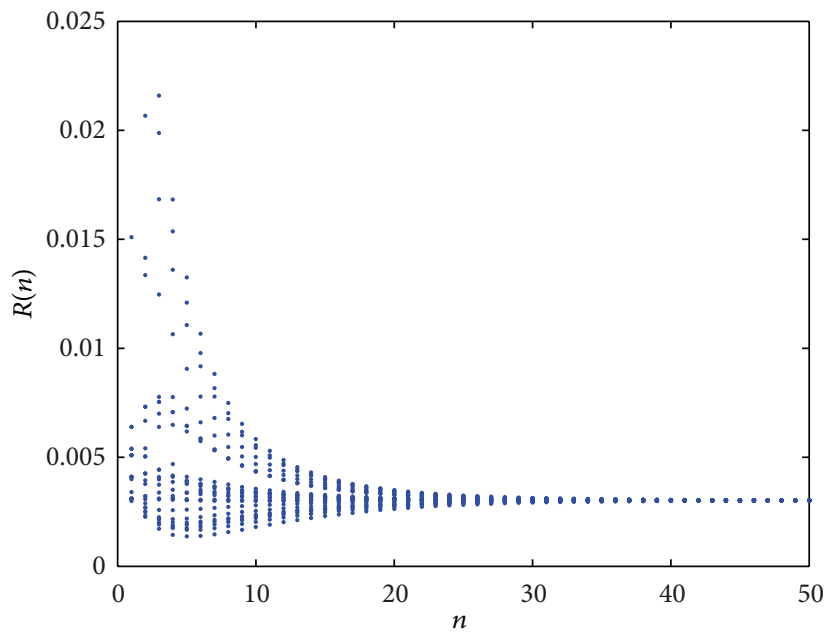

(c)

Figure 2: Time series of $S(n), I(n)$, and $R(n)$.

\section{Conclusions}

This paper deals with global stability of disease-free equilibrium and endemic equilibrium and the permanence of disease for a class of discrete SIRS epidemic models with nonlinear incidence rate $F(S) G(I)$ and disease-induced mortality. Under the basic assumption $\left(H_{1}\right)$, by applying analytic techniques, we obtain that disease-free equilibrium $E^{0}$ of model (1) is globally asymptotically stable if basic reproduction number $\mathscr{R}_{0} \leq 1$ and disease in the model is permanent if $\mathscr{R}_{0}>1$. Furthermore, motivated by the recent progress of Lyapunov techniques in continuous epidemic models (see, e.g., [25-27]), we construct the corresponding discrete analogue of Lyapunov functions (see Theorem 4) for nonlinear incidence rate $F(S) G(I)$. Under the assumptions burdened on $F(S)$, that is, assumptions $\left(H_{2}\right)$ and $\left(H_{3}\right)$, we prove that the global asymptotic stability for endemic equilibrium $E^{*}$ of model (1) for the case $\mathscr{R}_{0}>1$ is an extension of SIR-type models with nonlinear incidence rate $F(S) G(I)$ (see, for instance, $[7,26]$, etc.); that is, when SIRS models degenerate into SIR models, endemic equilibrium of the corresponding SIR models is globally asymptotically stable only if $\mathscr{R}_{0}>1$ and basic assumption $\left(H_{1}\right)$ hold.

From the proof of theorems in this paper, we easily see that discrete Lyapunov functions, such as $V(S, I, R)$ in Theorem 5, also can be applied for advanced models, including the models with delay. We expect to study the global stability of discrete SIRS and SEIRS epidemic models with rather general incidence rate $f(S, I)$ and with discrete or infinite delay, which is left as a future work.

\section{Conflict of Interests}

The authors declare that there is no conflict of interests regarding the publication of this paper. 


\section{Acknowledgments}

This work is supported by the National Natural Science Foundation of China (nos. 11271312 and 11201399), the Postdoctoral Science Foundation of China (Grant no. 20110491750), the Natural Science Foundation of Xinjiang (Grant nos. 2012211B07 and 2011211B08), and the Academic Discipline Project of Xinjiang Medical University Health Measurements and Health Economics (no. XYDXK50780308).

\section{References}

[1] P. van den Driessche and J. Watmough, "Reproduction numbers and sub-threshold endemic equilibria for compartmental models of disease transmission," Mathematical Biosciences, vol. 180, pp. 29-48, 2002.

[2] T. Zhang and Z. Teng, "Global behavior and permanence of SIRS epidemic model with time delay," Nonlinear Analysis, vol. 9, no. 4, pp. 1409-1424, 2008.

[3] T. Zhang and Z. Teng, "On a nonautonomous SEIRS model in epidemiology," Bulletin of Mathematical Biology, vol. 69, no. 8, pp. 2537-2559, 2007.

[4] Z. Teng, Y. Liu, and L. Zhang, "Persistence and extinction of disease in non-autonomous SIRS epidemic models with disease-induced mortality," Nonlinear Analysis, vol. 69, no. 8, pp. 2599-2614, 2008.

[5] Y. Nakata and T. Kuniya, "Global dynamics of a class of SEIRS epidemic models in a periodic environment," Journal of Mathematical Analysis and Applications, vol. 363, no. 1, pp. 230237, 2010.

[6] A. Korobeinikov and P. K. Maini, "A Lyapunov function and global properties for SIR and SEIR epidemiological models with nonlinear incidence," Mathematical Biosciences and Engineering, vol. 1, no. 1, pp. 57-60, 2004.

[7] A. Korobeinikov and P. K. Maini, "Non-linear incidence and stability of infectious disease models," Mathematical Medicine and Biology, vol. 22, no. 2, pp. 113-128, 2005.

[8] A. Korobeinikov, "Lyapunov functions and global stability for SIR and SIRS epidemiological models with non-linear transmission," Bulletin of Mathematical Biology, vol. 68, no. 3, pp. 615-626, 2006.

[9] A. Korobeinikov, "Global properties of infectious disease models with nonlinear incidence," Bulletin of Mathematical Biology, vol. 69, no. 6, pp. 1871-1886, 2007.

[10] A. Korobeinikov, "Global asymptotic properties of virus dynamics models with dose-dependent parasite reproduction and virulence, and nonlinear incidence rate," Mathematical Medicine and Biology, vol. 26, pp. 225-239, 2009.

[11] J. Li, Y. Xiao, F. Zhang, and Y. Yang, "An algebraic approach to proving the global stability of a class of epidemic models," Nonlinear Analysis, vol. 13, no. 5, pp. 2006-2016, 2012.

[12] C. Vargas-De-León, "On the global stability of SIS, SIR and SIRS epidemic models with standard incidence," Chaos, Solitons and Fractals, vol. 44, no. 12, pp. 1106-1110, 2011.

[13] C. Vargas-De-León, "Constructions of Lyapunov functions for classic SIS, SIR and SIRS epidemic models with variable population size," Revista Electrónica de Contenido Matemático, vol. 26 , no. 5, pp. 1-12, 2009.
[14] C. C. McCluskey, "Complete global stability for an SIR epidemic model with delay-distributed or discrete," Nonlinear Analysis, vol. 11, no. 1, pp. 55-59, 2010.

[15] C. C. McCluskey, "Global stability for an SEIR epidemiological model with varying infectivity and infinite delay," Mathematical Biosciences and Engineering, vol. 6, no. 3, pp. 603-610, 2009.

[16] C. C. McCluskey, "Global stability for an SIR epidemic model with delay and nonlinear incidence," Nonlinear Analysis, vol. 11, no. 4, pp. 3106-3109, 2010.

[17] V. Capasso and G. Serio, "A generalization of the KermackMcKendrick deterministic epidemic model," Mathematical Biosciences, vol. 42, no. 1-2, pp. 43-61, 1978.

[18] W. M. Liu, S. A. Levin, and Y. Iwasa, "Influence of nonlinear incidence rates upon the behavior of SIRS epidemiological models," Journal of Mathematical Biology, vol. 23, no. 2, pp. 187204, 1986.

[19] S. Ruan and W. Wang, "Dynamical behavior of an epidemic model with a nonlinear incidence rate," Journal of Differential Equations, vol. 188, no. 1, pp. 135-163, 2003.

[20] D. Xiao and S. Ruan, "Global analysis of an epidemic model with nonmonotone incidence rate," Mathematical Biosciences, vol. 208, no. 2, pp. 419-429, 2007.

[21] R. M. Cullen, A. Korobeinikov, and W. J. Walker, "Seasonality and critical community size for infectious diseases," The ANZIAM Journal, vol. 44, no. 4, pp. 501-512, 2003.

[22] Y. Enatsu, Y. Nakata, and Y. Muroya, "Global stability for a class of discrete SIR epidemic models," Mathematical Biosciences and Engineering, vol. 7, no. 2, pp. 347-361, 2010.

[23] Y. Muroya, A. Bellen, Y. Enatsu, and Y. Nakata, "Global stability for a discrete epidemic model for disease with immunity and latency spreading in a heterogeneous host population," Nonlinear Analysis, vol. 13, no. 1, pp. 258-274, 2012.

[24] Y. Enatsu, Y. Nakata, Y. Muroya, G. Izzo, and A. Vecchio, "Global dynamics of difference equations for SIR epidemic models with a class of nonlinear incidence rates," Journal of Difference Equations and Applications, vol. 18, no. 7, pp. 1163-1181, 2012.

[25] Y. Enatsu, Y. Nakata, and Y. Muroya, "Lyapunov functional techniques for the global stability analysis of a delayed SIRS epidemic model," Nonlinear Analysis, vol. 13, no. 5, pp. 21202133, 2012.

[26] G. Huang, Y. Takeuchi, W. Ma, and D. Wei, "Global stability for delay SIR and SEIR epidemic models with nonlinear incidence rate," Bulletin of Mathematical Biology, vol. 72, no. 5, pp. 1192$1207,2010$.

[27] G. Huang and Y. Takeuchi, "Global analysis on delay epidemiological dynamic models with nonlinear incidence," Journal of Mathematical Biology, vol. 63, no. 1, pp. 125-139, 2011.

[28] Z. Hu, Z. Teng, and H. Jiang, "Stability analysis in a class of discrete SIRS epidemic models," Nonlinear Analysis, vol. 13, no. 5, pp. 2017-2033, 2012.

[29] A. M. Lyapunov, The General Problem of The Stability of Motion, Taylor and Francis, London, UK, 1992.

[30] C. S. Peskin and T. Schlick, "Molecular dynamics by the backward-Euler method," Communications on Pure and Applied Mathematics, vol. 42, no. 7, pp. 1001-1031, 1989.

[31] T. Liu, C. Zhao, Q. Li, and L. Zhang, "An efficient backward Euler time-integration method for nonlinear dynamic analysis 
of structures," Computers and Structures, vol. 106, pp. 20-28, 2012.

[32] L. Wang and M. Wang, Ordinary Difference Equation, Xinjiang University Press, Xinjiang, China, 1989 (Chinese).

[33] J. P. LaSalle, The Stability of Dynamical Systems, Society for Industrial and Applied Mathematics, Philadelphia, Pa, USA, 1976.

[34] X. Zhao, Dynamical Systems in Population Biology, Springer, New York, NY, USA, 2003. 


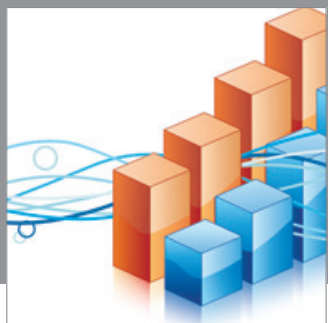

Advances in

Operations Research

mansans

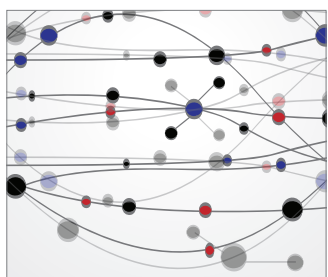

The Scientific World Journal
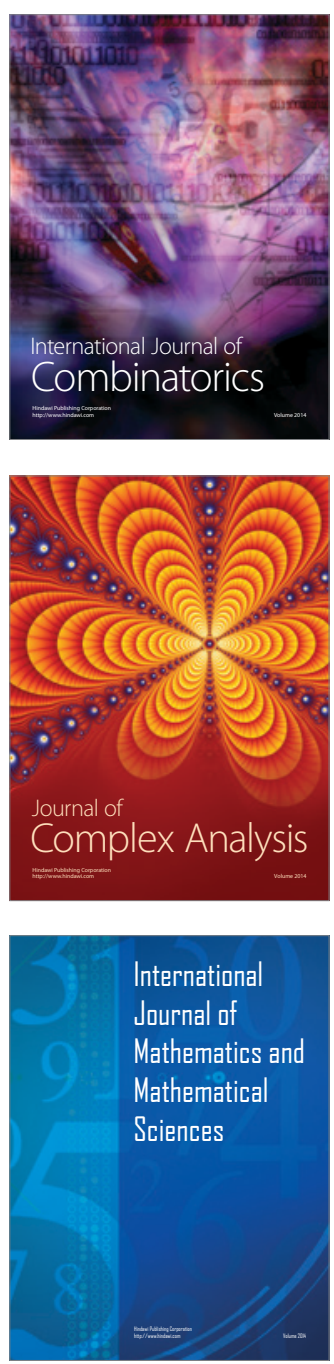
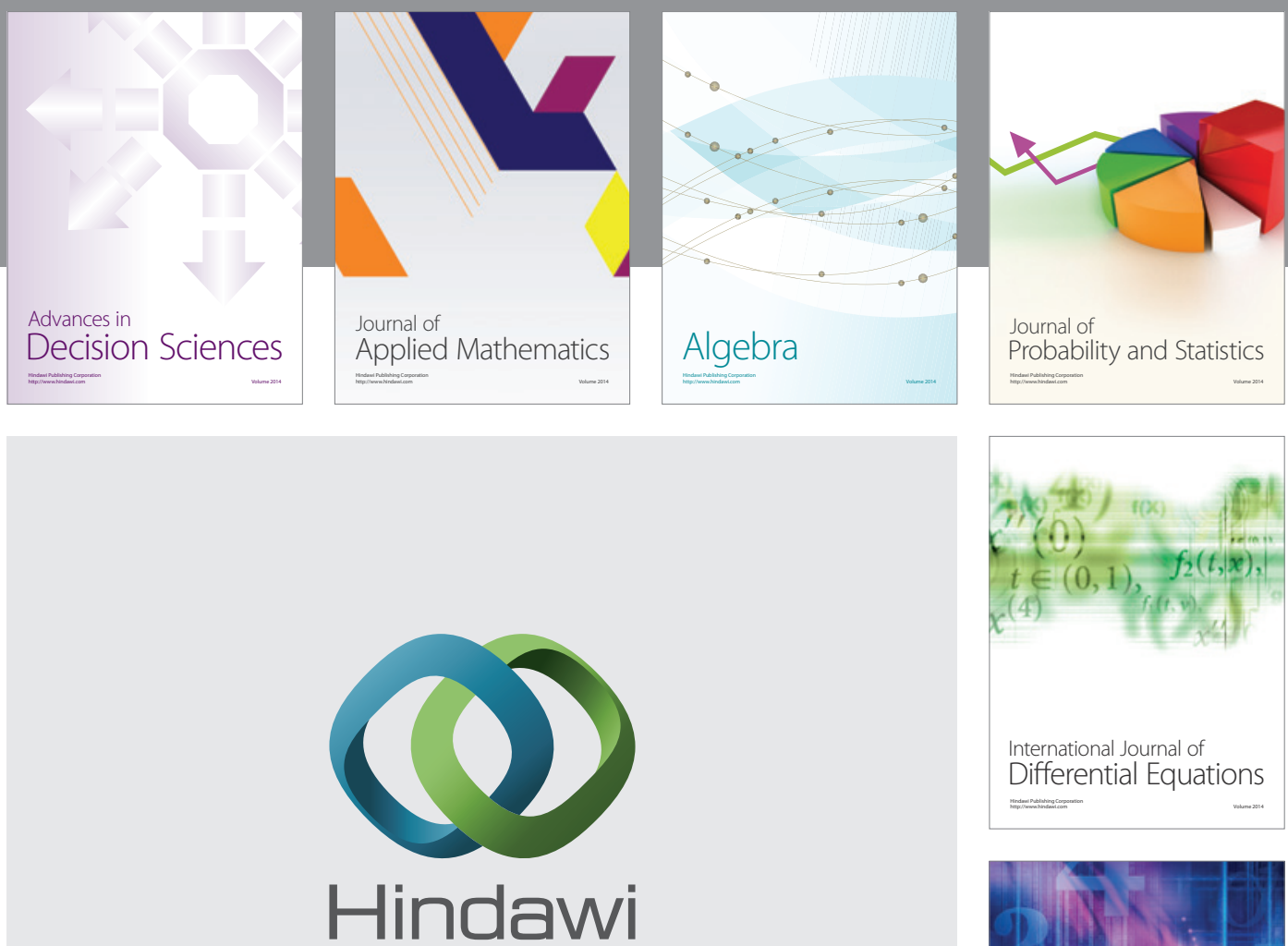

Submit your manuscripts at http://www.hindawi.com
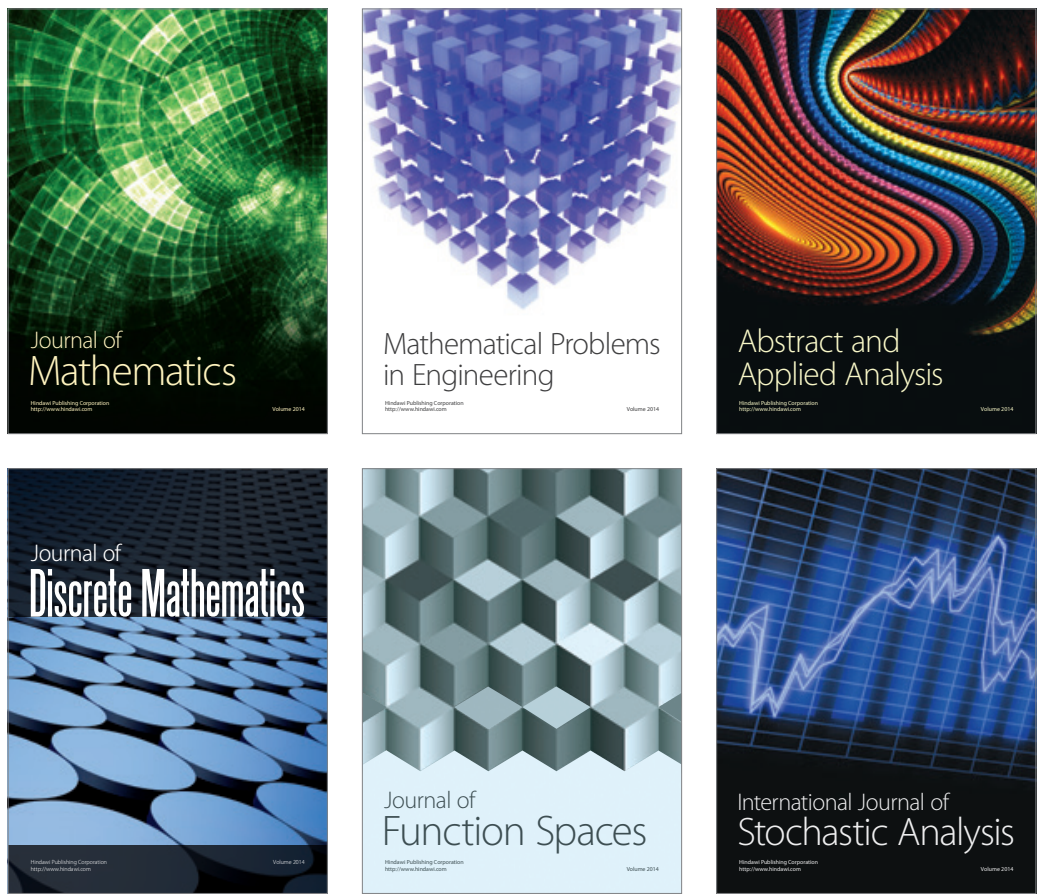

Journal of

Function Spaces

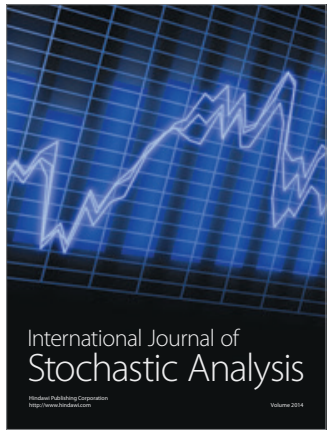

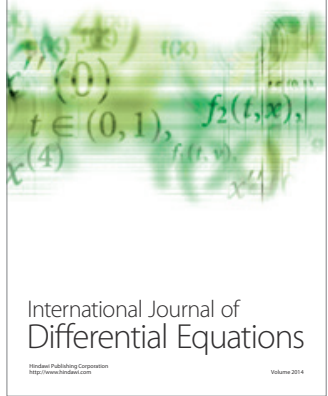
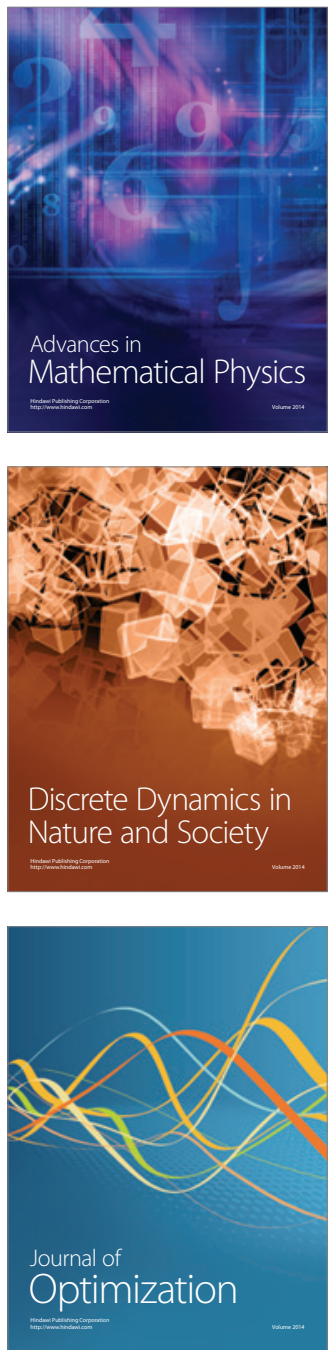